Published in final edited form as:

Nat Genet. 2019 November ; 51(11): 1588-1595. doi:10.1038/s41588-019-0524-6.

\title{
The impact of pro-inflammatory cytokines on the $\beta$-cell regulatory landscape provides insights into the genetics of type 1 diabetes
}

\author{
Mireia Ramos-Rodríguez ${ }^{1}$, Helena Raurell-Vila ${ }^{1}$, Maikel L. Colli², Maria Inês Alvelos ${ }^{2}$, Marc \\ Subirana-Granés ${ }^{1}$, Jonàs Juan-Mateu ${ }^{2}$, Richard Norris ${ }^{1}$, Jean-Valery Turatsinze ${ }^{2}$, Ernesto \\ S. Nakayasu ${ }^{3}$, Bobbie-Jo M. Webb-Robertson ${ }^{3}$, Jamie R.J. Inshaw ${ }^{4}$, Piero Marchetti ${ }^{5}$, \\ Lorenzo Piemonti ${ }^{6}$, Manel Esteller ${ }^{7,8,9,10}$, John A. Todd ${ }^{4}$, Thomas O. Metz ${ }^{3}$, Décio L. \\ Eizirik $^{2}$, Lorenzo Pasquali ${ }^{1,7,11}$ \\ ${ }^{1}$ Endocrine Regulatory Genomics Laboratory, Germans Trias i Pujol University Hospital and \\ Research Institute, Badalona, Spain. \\ ${ }^{2}$ ULB Center for Diabetes Research and Welbio, Medical Faculty, Université Libre de Bruxelles, \\ Brussels, Belgium. \\ ${ }^{3}$ Biological Sciences Division, Pacific Northwest National Laboratory, Richland, WA, USA. \\ ${ }^{4}$ JDRF/Wellcome Diabetes and Inflammation Laboratory, Wellcome Centre for Human Genetics, \\ Nuffield Department of Medicine, NIHR Oxford Biomedical Research Centre, University of Oxford, \\ Roosevelt Drive, Oxford, UK. \\ ${ }^{5}$ Department of Clinical and Experimental Medicine, University of Pisa, Pisa, Italy. \\ ${ }^{6}$ Diabetes Research Institute (HSR-DRI), San Raffaele Scientific Institute, Milan, 20132, Italy. \\ 7Josep Carreras Leukaemia Research Institute (IJC), Badalona, Barcelona, Catalonia, Spain. \\ ${ }^{8}$ Centro de Investigacion Biomedica en Red Cancer (CIBERONC), Madrid, Spain \\ ${ }^{9}$ Institució Catalana de Recerca i Estudis Avançats (ICREA), Barcelona, Catalonia, Spain. \\ ${ }^{10}$ Physiological Sciences Department, School of Medicine and Health Sciences, University of \\ Barcelona (UB), Barcelona, Catalonia, Spain. \\ ${ }^{11}$ CIBER de Diabetes y Enfermedades Metabólicas Asociadas (CIBERDEM), Barcelona, Spain.
}

\begin{abstract}
Users may view, print, copy, and download text and data-mine the content in such documents, for the purposes of academic research, subject always to the full Conditions of use:http://www.nature.com/authors/editorial_policies/license.html\#terms

*Corresponding author: LP: 1pasquali@igtp.cat.

Author contributions:

L.P., M.R. and D.E. designed the experiments. H.R., M.C., M.A., J.J., R.N. and E.N. performed and analyzed the experiments. M.R. performed bioinformatic analyses with contribution from M.S. J-V.T., B.W. and J.I.. L.Pi., P.M., M.E., and T.M. provided material and resources. L.P., D.L.E., T.M. and J.A.T.. supervised the study. LP and MR coordinated, conceived the project and wrote the manuscript with contribution from D.E. All authors reviewed the final manuscript.

Competing Interests:

Authors declare no conflict of interest.
\end{abstract}


Early stages of type 1 diabetes (T1D) are characterized by local autoimmune inflammation and progressive loss of insulin-producing pancreatic $\beta$ cells. We show here that exposure to proinflammatory cytokines unmasks a marked plasticity of the $\beta$-cell regulatory landscape. We expand the repertoire of human islet regulatory elements by mapping stimulus-responsive enhancers linked to changes in the $\beta$-cell transcriptome, proteome and 3D chromatin structure. Our data indicate that the $\beta$ cell response to cytokines is mediated by the induction of new regulatory regions as well as the activation of primed regulatory elements prebound by isletspecific transcription factors. We find that T1D-associated loci are enriched of the newly mapped cis-regulatory regions and identify T1D-associated variants disrupting cytokine-responsive enhancer activity in human $\beta$ cells. Our study illustrates how $\beta$ cells respond to a proinflammatory environment and implicate a role for stimulus-response islet enhancers in T1D.

In type 1 diabetes (T1D), early inflammation of the pancreatic islets (insulitis) by $\mathrm{T}$ and $\mathrm{B}$ cells contributes to both the primary induction and secondary amplification of the immune assault, with inflammatory mediators such as the cytokines interleukin-1 $\beta$ (IL-1 $\beta$ ) and interferon- $\gamma$ (IFN- $\gamma$ ) contributing to the functional suppression and apoptosis of $\beta$ cells $^{1-3}$.

Genome wide association studies (GWAS) have made a substantial contribution to the knowledge of T1D genetic architecture uncovering $>60$ regions containing thousands of associated genetic variants. Nevertheless, translating variants to function remains a main challenge for T1D and other complex diseases. Most of the associated variants do not reside in coding regions ${ }^{4}$, suggesting that they may influence transcript regulation rather than altering protein coding sequences. Recent studies showed a primary enrichment of T1D association signals in $\mathrm{T}$ and $\mathrm{B}$ cell enhancers ${ }^{4,5}$. A secondary ${ }^{5}$, or a lack of enrichment, was instead observed in islet regulatory regions. While such observation points to a major role of the immune system, we hypothesize that a subset of T1D variants may also act at the $\beta$-cell level but only manifest upon islet-cell perturbation and are thus not captured by the current maps of islet regulatory elements.

We have now mapped inflammation-induced cis-regulatory networks, transcripts, proteins and 3D chromatin structure changes in human $\beta$ cells (Fig. 1a). We leverage these data to unmask functional T1D genetic variants as well as key candidate genes and regulatory pathways contributing to the $\beta$ cell autoimmune destruction. Such analyses permit elucidation of the role of epigenetic gene regulation and its interaction with T1D genetics in the context of the autoimmune reaction that drives $\beta$ cell death.

\section{Results}

\section{Pro-inflammatory cytokines impact the $\beta$-cell chromatin landscape.}

To characterize the effect of pro-inflammatory cytokines on the $\beta$-cell regulatory landscape, we first mapped all accessible or open chromatin sites in human pancreatic islets exposed or not to IFN- $\gamma$ and IL-1 $\beta$. We assayed chromatin accessibility by ATAC-seq and, in order to focus on the $\beta$-cell fraction and to decrease inter-individual variability, in parallel with human pancreatic islet assays, we performed ATAC-seq in the clonal human $\beta$-cell line EndoC- $\beta \mathrm{H} 1^{6}$, exposed or not to the pro-inflammatory cytokines (overall number of peaks 
identified in human islets: 92,610-229,588; and in EndoC- $\beta$ H1 cells: 52,735-110,715.

Extended Data Fig. 1a). Such experiments unmasked an important remodeling of the $\beta$-cell chromatin resulting in $\sim 12,500$ high confident chromatin sites that gain accessibility (FDR adjusted $P<0.05 ; \log _{2}$ FC $>1$ ) (Extended Data Fig. 1b) upon exposure to pro-inflammatory cytokines. Importantly, the changes observed in the human $\beta$ cell line were concordant with those observed in the human islet preparations (Extended Data Fig. 1c).

We reasoned that changes in chromatin accessibility may reflect the activation of non-coding cis-regulatory elements. We thus used chromatin immunoprecipitation coupled with next generation sequencing (ChIP-seq) to map cytokine-induced changes of H3K27ac (Extended Data Fig. 1a), a key histone modification associated with active cis-regulatory elements that was shown to be dynamically regulated in response to acute stimulation ${ }^{7}$. We observed genome-wide deposition of the active histone modification mark upon exposure to proinflammatory cytokines in both EndoC- $\beta \mathrm{H} 1$ and human pancreatic islets (Extended Data Fig. 1b-c).

Integrative analysis of ATAC-seq and ChIP-seq indicates that changes in chromatin accessibility are strongly correlated with deposition of H3K27ac $\left(P<2 \times 10^{-16}, \mathrm{r}^{2}=0.63\right)$ allowing the identification of $\sim 3,800$ open chromatin regions that gained H3K27ac (FDR adjusted $P<0.05 ; \log _{2} \mathrm{FCl}>1$ ) upon exposure to pro-inflammatory cytokines (Fig. $1 \mathrm{~b}$ and Extended Data Fig. 1d). We found that this subset of open chromatin regions is preferentially located distally to gene transcription start sites (TSS) (Extended Data Fig. 1e), their sequence is evolutionary conserved (Extended Data Fig. 1f) and enriched for specific transcription factor (TF) binding sites (Extended Data Fig. 1g). We named these newly mapped regions IREs for “Induced Regulatory Elements" (Supplementary Table 1 and Supplementary Table 2).

\section{Chromatin changes link to variation in transcription and translation.}

We next explored whether the newly identified IREs were associated with changes in gene expression and protein translation. To identify $\beta$ cell transcripts and proteins induced by the pro-inflammatory cytokines we assayed gene expression by RNA-seq (five replicates in EndoC- $\beta \mathrm{H} 1$ and five replicates in human pancreatic islets ${ }^{8}$, Extended Data Fig. 1a) and collected multiplex proteomics data for three EndoC- $\beta \mathrm{H} 1$ replicates after exposure or not to pro-inflammatory cytokines.

In line with the chromatin assays, that indicated extensive gene regulatory activation, we unraveled cytokine-induced transcriptional activation resulting in $~ 1,200$ upregulated genes (FDR adjusted $P<0.05 ; \log _{2} \mathrm{FC}>1$ ) (Extended Data Fig. 2a-b). By multiplex proteomics, after rigorous filtering, a subset of 10,166 proteins was confidently quantified and retained for significance testing. A total of 348 proteins displayed significant changes in abundance (FDR/Q-value $<0.15$ and $|\mathrm{FCl}>1.5 ;| \log _{2} \mathrm{FCl}>0.58$ ) being $2.19 \%$ of the overall detected proteins upregulated (Extended Data Fig. 2c), 76\% of which had induced mRNA levels at $48 \mathrm{~h}$, confirming consistency between RNA-seq and protein changes $\left(\mathrm{r}^{2}=0.72, P<2 \times 10^{-16}\right)$ (Fig. 1c). Protein-protein interactions inferred from $\beta$-cell cytokine-induced proteins resulted in a network more connected than expected by chance $\left(P<10^{-3}\right)$, significantly enriched for Molecular Signatures Database (MSigDB; http://software.broadinstitute.org/gsea/msigdb/) 
pathways including IFN- $\gamma$ signaling, antigen processing and presentation, apoptosis and T1D (KEGG T1D $P=7.9 \times 10^{-8}$, Extended Data Fig. 2d).

In line with our expectations we found that IREs were linked to up-regulation of the nearby gene/s as well as to an induced abundance of the corresponding protein (Fig. 1d-e and Extended Data Fig. 2e). Moreover, gene induction was highly correlated with the number of associated IREs, suggesting a cumulative effect of IREs on cytokine-induced changes in gene expression (Extended Data Fig. 2f).

Taken together these findings reveal that pancreatic $\beta$-cell response to pro-inflammatory cytokines is dynamic, involving extensive chromatin remodeling and profound changes in the regulatory landscape (Fig. If and Extended Data Fig. 2g). Such changes are associated with induction of transcription and protein translation including pathways implicated in the pathogenesis of T1D. Newly defined regulatory maps can be visualized online along with other islet regulatory annotations at www.isletregulome.org.

\section{Primed and neo regulatory elements mediate cytokine response.}

We next sought to gain insight into the dynamic activation of IREs. The relationship between chromatin openness and $\mathrm{H} 3 \mathrm{~K} 27 \mathrm{ac}$ deposition upon exposure to pro-inflammatory cytokines allows the distinction of two classes of IREs (Fig. 1b and Fig. 2a-c): "opening IREs" (n=2,436) which gain both chromatin accessibility $\left(\log _{2} \mathrm{FC}>1\right)$ and $\mathrm{H} 3 \mathrm{~K} 27 \mathrm{ac}\left(\log _{2}\right.$ $\mathrm{FC}>1)$; and "primed IREs" $(\mathrm{n}=1,362)$ which are already accessible chromatin sites prior to the treatment (ATAC-seq $\log _{2} \mathrm{FC}<1$ ) and gain $\mathrm{H} 3 \mathrm{~K} 27 \mathrm{ac}\left(\log _{2} \mathrm{FC}>1\right)$ upon exposure to the stimulus. Primed and opening IREs are both associated to gene expression induction (Extended Data Fig. 3a), phylogenetically conserved (Extended Data Fig. 3b) and preferentially mapped distally relatively to gene TSS (Extended Data Fig. 3c). We further unmasked that $70 \%$ of opening IREs $(n=1,716)$ are newly activated (i.e. undetectable in basal condition, see methods). We named the latter "neo IREs". Neo IREs represent $45 \%$ of all IREs and may mirror "latent enhancers" that were identified upon stimulation of mouse macrophages ${ }^{7}$.

Because chromatin openness, the feature discerning the two classes of IREs, is believed to reflect TF occupancy, we analyzed their sequence composition in search of recognition sequences of key TFs orchestrating the $\beta$ cell response to pro-inflammatory cytokines. Even though IREs are mostly distal to TSS (Extended Data Fig. 3c), in order to reduce sequence biases, we excluded from this analysis all annotated promoters. The two classes of distal IREs predominantly mapped to enhancer chromatin state (Extended Data Fig. 3d) and showed clear differences in sequence composition. Newly induced enhancers were enriched for binding motifs of inflammatory-response TFs including Interferon-Sensitive Response Element (ISRE), STAT and NF-kB (Extended Data Fig. 3e). Primed enhancers instead were enriched for binding motifs of inflammatory-response TFs (ISRE, STAT), and unexpectedly, islet-specific TFs (HNF1A/B, NEUROD1, PDX1, MAFB, NKX6.1) (Extended Data Fig. 3f). Importantly, we found that, in primed enhancers, inflammatory-response and isletspecific TFs binding motifs mapped to the same genomic region, suggesting co-binding and possibly cooperation of the two classes of TFs (Extended Data Fig. 3g-h). 
Sequence composition bias per se does not imply TF occupancy. We thus took advantage of published ChIP-seq datasets of islet-specific TFs (MAFB, PDX1, FOXA2, NKX6.1 and NKX2.2) mapped in un-stimulated human pancreatic islets ${ }^{9}$ to measure TF occupancy in primed and neo enhancers prior to the pro-inflammatory stimulus. As expected from the sequence composition analysis, primed enhancers (unlike neo enhancers) are highly bound by tissue-specific TFs even before their activation (Fig. 2d and Extended Data Fig. 3i). TF occupancy can also be indirectly assessed by ATAC-seq, which assays the protection of the bound sequence to transposase cleavage (footprint). Footprint analysis is effective for TFs with a long residence time ${ }^{10}$ such as IRFs and STAT TF families. Our analyses revealed the emergence of footprint marks upon pro-inflammatory treatment in correspondence to ISRE motifs in both primed and neo enhancers (Fig. 2e) indicating cytokine-induced TF occupancy of IREs.

Gene regulation is orchestrated by different epigenetics mechanisms. DNA methylation is a relatively stable epigenetic mark contributing to maintenance of cellular identity ${ }^{11,12}$. Moreover, high-resolution DNA methylation maps, obtained from multiple tissues, established that the vast majority of tissue-specific differentially methylated regions are located at distal, mostly non-coding, regulatory sites ${ }^{13}$. Consequently, characterization of the DNA methylome in the context of relevant stimuli is important for understanding the functional mechanisms of tissue-specific responses in human disease ${ }^{14}$. We thus explored if cytokine-induced chromatin remodeling is associated with changes in DNA methylation. We quantified DNA methylation changes by performing dense methylation arrays in EndoC$\beta \mathrm{H} 1$ exposed or not to IFN- $\gamma$ and IL-1 $\beta$. The Infinium MehtylationEPIC array was designed to interrogate with high precision and coverage $>850,000 \mathrm{CpG}$ sites (approximately $3 \%$ of all sites in the genome) selected primarily because of their location close to gene promoters and CpG-island regions. By focusing on the 1,230 IRE enhancers harboring one or more CpG sites interrogated by the array, we observed that primed enhancers overlap lowly methylated CpGs (median $\beta$-value $0.12 \pm 0.08$ ), that did not vary significantly upon cytokine exposure. Such observation is in sharp contrast with neo enhancers that were highly methylated under control condition (median $\beta$-value $0.77 \pm 0.10$ ) but underwent a significant loss of DNA methylation (Two-sided Wilcoxon test, $P=4.13 \times 10^{-4}$ ) upon the treatment (Fig. $2 \mathrm{f}$ ). While we did not observe cytokine-induced methylation, we found that $\sim 70 \%$ of the significantly demethylated probes (FDR adjusted $P \unlhd$ ).05; $\beta_{\text {cyt }}-\beta_{\text {ctrl }}<-0.20$ ) mapping to IRE were located at neo enhancers (Extended Data Fig. 3j,k)

These results suggest that neo enhancers are enriched for methylated CpGs that undergo preferential demethylation upon cytokine treatment whereas primed enhancers are enriched for unmethylated $\mathrm{CpGs}$ that do not change their methylation status upon the cytokine exposure.

Taken together these analyses lead to a model, in which pro-inflammatory cytokines elicit a regulatory response in $\beta$ cells characterized by: 1) new induction of distal regulatory elements coupled with reduction of DNA methylation and binding of inflammatory response TFs and 2) activation of regulatory elements pre-bound by islet-specific TFs and induced by inflammatory response TFs (Fig. 2g). 
Collectively, these results allow reconstructing cis-regulatory networks activated in human pancreatic $\beta$ cells upon exposure to the pro-inflammatory cytokines IFN- $\gamma$ and IL-1 $\beta$ (Extended Data Fig. 4a-c and Supplementary Table 1).

\section{Changes in the islet 3D chromatin structure.}

Regulatory regions can exert control over genes at megabase distances through the formation of DNA loops. These loops are often confined within structures known as topologically associating domains (TADs) ${ }^{15-17}$. TADs are largely conserved upon evolution, are invariant in different cell types and have their boundaries defined by the regulatory scope of tissuespecific enhancers ${ }^{18-19}$. Our knowledge regarding the general characteristics and mechanisms of loops is improving ${ }^{20-23}$, but much less is known regarding mechanisms and functional significance of dynamic looping events during biological processes.

We took advantage of promoter capture Hi-C (pcHi-C) performed in human pancreatic islets ${ }^{24}$ to explore long-range interactions between gene promoters and cytokine-induced and invariant distant regulatory elements. Interestingly, we observed that the interaction confidence scores captured between IRE enhancers and gene promoters in untreated islets were significantly reduced compared with SREs enhancers $\left(P=1.8 \times 10^{-11}\right)$ (Extended Data Fig. 5a). As this finding points to potential dynamic properties of the interaction maps, we next sought to investigate if cytokine-induced regulatory changes are linked to modification of the 3D chromatin structure and if induction of $\beta$-cell cytokine-responsive regulatory elements is coupled with the formation of novel DNA looping interactions.

Hi-C profiles are limited in sequencing coverage and library complexity, resulting in maps of reduced resolution relative to regulatory maps of functional elements. On the other hand, $4 \mathrm{C}$ approaches are difficult to interpret quantitatively mainly due to potential amplification biases. We thus applied targeted chromosome capture with unique molecular identifiers (UMI-4C), a recently developed method ${ }^{25}$, to quantitatively measure interaction intensities in human islets before and after exposure to pro-inflammatory cytokines. We centered the conformation capture viewpoint to the promoter of 13 genes (TNFSF10, GBP1, CIITA, among others) whose expression was strongly induced by the cytokine exposure.

UMI-4C showed marked changes in the 3D chromatin structure at the analyzed loci. Promoters of the induced genes gained chromatin interactions with distal genomic regions reflecting the formation of new DNA looping events (Fig. 3a-b and Extended Data Fig. 5bd). Importantly, such new contacts were preferentially engaged with newly mapped human islet cytokine-responsive IREs (Fig. 3c).

These results demonstrate that cytokine exposure induces changes in human islet 3D chromatin conformation including the formation of novel enhancer-promoter interactions. Such changes allow the newly activated distal IREs to contact their target gene promoters.

\section{Islet cytokine enhancers are implicated in T1D genetic susceptibility.}

GWAS have identified $\sim 60$ chromosome regions associated with $\mathrm{T}^{2} \mathrm{D}^{26}$ with many of the association signals having been assigned to candidate genes with immunological functions. Consistent with this notion, several studies reported a primary enrichment of T1D risk 
variants in $\mathrm{T}$ and $\mathrm{B}$ cell regulatory elements ${ }^{4,5}$. Furthermore, there is a substantial lack of statistical significant overlap of T1D associated variants in islet enhancers, while such regulatory elements are instead enriched for GWAS signals for T2D and fasting glucose ${ }^{9,27}$. Nonetheless, the molecular mechanisms linking T1D association signals to cellular functions remain poorly described for most of the regions of association identified.

We hypothesized that a subset of T1D genetic signals may reflect an altered capacity of the $\beta$ cells to react to an inflammatory environment. We thus sought to explore to what extent genetic signals underlying T1D susceptibility act through pancreatic islet regulatory response to pro-inflammatory cytokines.

Causal cis variants are expected to lie in sequences that act as regulatory regions in statespecific and disease-relevant tissues. We thus examined non shared loci with genome-wide significant association to T2D and T1D in European populations and considered all variants in high LD (1000 Genomes Project phase3 EUR $\left.\mathrm{R}^{2}>0.8\right)$ with a lead SNP reported in the NHGRI-EBI GWAS catalog ${ }^{26}$. In line with previous observations ${ }^{4,9}$, we found that T2D but not T1D risk variants overlap human islet non cytokine-responsive regulatory elements (i.e. SREs) more than expected by chance (T2D SNPs in SREs $P<2 \times 10^{-16}, \mathrm{Z}=5.47$ ). In contrast, we uncovered that human islet IREs are enriched for T1D but not T2D risk variants (T1D SNPs in IREs $P=3 \times 10^{-6}, \mathrm{Z}=4.61$ ) (Fig. $4 \mathrm{a}$ ). This result was reproduced when using regulatory elements detected in EndoC- $\beta \mathrm{H} 1$ cells (Extended Data Fig. 6a). Such findings unmasked 9 T1D associated regions (13\% of the total) containing at least one islet cytokineinduced regulatory element directly overlapping a T1D associated variant (Supplementary Table 3 and Extended Data Fig. 6b-f).

We noticed that the two T1D lead SNPs at 1q24.3 and 16q13.13 loci (rs78037977 28,29 and rs $193778^{4}$ respectively) were directly overlapping IREs in islets. We used GWAS genotyping data from a cohort of 14,575 individuals (5,909 T1D cases and 8,721 controls, see methods) to confirm their association with T1D. Both variants were included in the $99 \%$ credible set of their respective locus and displayed strong association $P$-values (rs 78037977 $P=6.94 \mathrm{e}^{-10} ;$ rs $193778 P=1.33 \mathrm{e}^{-7}$; see Supplementary Table 4 for posterior probability of association and variant ranking in the credible set), indicating that they could potentially be causal.

At the 1q24.3 locus, rs78037977 (NC_000001.10:g.172715702A>G) overlaps an islet cytokine-induced chromatin site (Fig. 4b) which is pre-bound by islet-specific TFs and is a predicted enhancer in other cell types (Extended Data Fig. 6g). We created allele-specific luciferase reporter constructs and measured enhancer activity in the EndoC- $\beta \mathrm{H} 1$ line before and after cytokine exposure. The sequence exerts enhancer activity exclusively after cytokine exposure which is disrupted by the rs78037977, T1D associated, $G$ allele (One-way ANOVA, $\mathrm{F}=26, P=4.34 \times 10^{-5}$ ) (Fig. $4 \mathrm{c}$ and Extended Data Fig. $6 \mathrm{~h}$ ) consistent with a causal role of the variant at this locus. In order to identify the gene target of this T1D-susceptible enhancer, we reconstructed the 3D chromatin structure by chromatin capture experiments. UMI-4C in human islets identified a cytokine-induced interaction of the enhancer with TNFSF18 a gene activated in islets upon cytokine exposure (Fig. 4d-e). TNFSF18 encodes for a cytokine, ligand of the TNFRSF18/GITR receptor, known to modulate inflammatory 
reaction and regulation of autoimmune responses ${ }^{30}$. Interestingly, we noticed that cytokine exposure results in upregulation of TNFSF18 in human islets but not in the EndoC- $\beta \mathrm{H} 1 \beta$ cell line, suggesting differences in gene regulatory dynamics in primary tissue or the activation of an islet cell sub-population.

At the 16q13.13 locus, rs193778 (NC_000016.9:g.11351211A>G) maps to a phylogenetically conserved, cytokine-responsive regulatory element (Fig. 4f). This sequence displays enhancer activity in both treated and untreated $\beta$ cells. However, exclusively in cytokine-exposed $\beta$ cells, the T1D-associated $G$ allele exerts significantly higher enhancer activity than the protective variant (One-way ANOVA, $\mathrm{F}=12.34, P=1.23 \times 10^{-3}$ ) (Fig. $4 \mathrm{~g}$ and Extended Data Fig. 6i). The locus includes several up-regulated genes (SOCS1, DEXI, CIITA, RMI2), that could represent potential targets of this IRE. Recent works point to $D E X I$ as a T1D candidate gene in immune cells and $\beta$ cells ${ }^{31,32}$. By performing UMI-4C experiments in human islets we observed a strong chromatin contact between the promoter of $D E X I$ and the regulatory element bearing the rs193778 T1D-associated variant (Fig. 4h). Such data points to $D E X I$ as a potential causal gene in pancreatic islets.

Altogether these results illustrate how unraveling cytokine-induced chromatin dynamics in human islets can guide the identification of cis-regulatory variants that are strong candidates in driving T1D-association signals.

\section{Discussion:}

Our work illustrates the human pancreatic $\beta$ cell chromatin dynamics in response to an external stimulus that may be relevant in the context of T1D. We here show that exposure to pro-inflammatory cytokines causes profound remodeling of the $\beta$-cell regulatory landscape coupled with changes in gene expression and protein production. The degree of the regulatory network remodeling was comparable to that previously shown for macrophages or mouse dendritic cells exposed to similar stimuli ${ }^{7}$. We unveil the activation of $\sim 3,600$ cytokine-responsive distal cis regulatory elements and reveal a lack of homogeneity in their molecular mechanism of activation. We observed that the induction of a subset of novel regulatory regions (neo IREs) require TF binding and chromatin opening while other chromatin sites are "primed" to their activation being pre-bound by islet-specific TFs. Our observations suggest a model in which binding of tissue-specific TFs may facilitate chromatin accessibility at a subset of chromatin sites that can then be promptly activated by the induction of inflammatory-response TFs. Such model is supported by very recent findings ${ }^{33}$ and consistent with observations in murine macrophages 7,34 and murine dendritic cells ${ }^{35}$, but thus far had not been demonstrated in a highly differentiated and non-immune related tissue such as the pancreatic islets. Even though our model suggests that exposure to proinflammatory cytokines causes predominantly induction of gene transcription rather than transcript down-regulation, we cannot exclude that a more prolonged stimulus could induce loss of critical $\beta$ cell processes resulting from the reduction of $\beta$ cell cis-regulatory networks activity.

Importantly, we show that such regulatory changes are coupled with 3D chromatin remodeling, allowing the newly activated regulatory elements to contact their target genes. 
Several reports described 3D chromatin dynamics properties in the cell developmental context $^{36,37}$, upon loss of cell fate ${ }^{38,39}$, senescence ${ }^{40,41}$ or in response to hormonal exposure $^{42}$. Our observations indicate that the capacity of enhancer loop formation is maintained in a highly-differentiated tissue such as the islets and it is coupled with transcriptional regulatory changes, in response to an external stimulus.

The model used in our study to explore chromatin dynamics is of particular interest because it mimics the inflammatory environment that the pancreatic islets may face in the early stages of T1D. While several T1D candidate genes regulating key steps related to "danger signal recognition" and innate immunity were shown to be expressed in human islets ${ }^{43}$, T1D associated variants were shown to be enriched for immune cell types but not in stable pancreatic islets regulatory elements ${ }^{4}$. Such apparent contradiction may be reconciled by our findings showing that human islet cytokine-responsive regulatory elements are enriched for T1D risk variants. Our data, supported by recent findings unmasking regulatory variants affecting enhancer activation in immune response $\mathrm{e}^{33,44}$, opens the avenue to identify T1D molecular mechanisms acting at the pancreatic islet cells level.

Although we cannot exclude that functional variants disrupting $\beta$-cell regulatory mechanisms may at the same time affect the regulatory potential of immune-related cell types, the availability of stimulus-responsive cis-regulatory maps in pancreatic islets will facilitate hypothesis-driven experiments to uncover how common and lower frequency genetic variants impact islet cells in T1D. We here studied the human islet responses to a specific pro-inflammatory stimulus. Future work, studying additional immune-mediated stresses potentially affecting $\beta$ cells at different stages of the disease, may allow uncovering other association signals acting at the islet cell level.

In general, our findings could apply by extension to other diseases where "primed" enhancers may facilitate cell-type-specific responses to ubiquitous signals resulting in tissuespecific genetic susceptibility in autoimmune diseases.

\section{Methods:}

\section{Human Islets and EndoC- $\beta \mathrm{H} 1$}

Human islets from 14 multiorgan donors without a history of glucose intolerance were isolated in compliance with ethical regulations (Supplementary Note 1) and according to established isolation procedures ${ }^{45,46}$ (Supplementary Note 2 and Supplementary Table 5). The human insulin-producing EndoC- $\beta \mathrm{H} 1$ cells where kindly provided by $\mathrm{Dr}$ R. Scharfmann, University of Paris, France ${ }^{6}$ and cultured in DMEM medium (Supplementary Note 3).

Human islets and EndoC- $\beta \mathrm{H} 1$ cells where exposed or not to a cocktail of pro-inflammatory cytokines IFN- $\gamma$ and IL- $1 \beta$ for 48 hours. The cytokine concentrations used were those described in previous dose-response experiments ${ }^{47-49}$ (Supplementary Note 2). The glucose stimulation index was tested on human islet preparations and EndoC- $\beta \mathrm{H} 1$ samples to confirm functional competence of the samples (Supplementary Note 4 and Extended Data Fig. 7). 


\section{ChIP-seq and ATAC-seq}

ATAC-seq library preparations were carried out as previously described ${ }^{50}$ with minor modifications ${ }^{51,52}$ (Supplementary Note 5). ChIP-seq was carried out using tagmentation (ChIPmentation) as previously described ${ }^{53}$ (Supplementary Note 6).

ATAC-seq and ChIP-seq libraries were sequenced on Illumina HiSeq 2500. Reads were aligned to the hg 19 reference genome using Bowtie 2 (version 2.3.4.1) ) $^{54}$ using default parameters. After alignment, reads mapping to ENCODE blacklist regions ${ }^{55}$, to noncanonical chromosomes or to mitochondrial DNA were discarded. Duplicates were removed using samtools markdup (version 1.8) ${ }^{5656}$ (see Supplementary Table 6 for number of mapped reads per experiment and Extended Data Fig. 8 for measures of ATAC-seq quality).

Peaks were called with MACS2 callpeak (version 2.1) ${ }^{57}$ with parameters "-q 0.05 --nomodel --shift -100 --extsize 200” for ATAC-seq and “--broad --broad-cutoff 0.1 --nomodel” for H3K27ac ChIP-seq. A more in-detail description of the bioinformatics processing can be found in Supplementary Note 7.

\section{RNA-seq}

Total RNA was isolated from EndoC- $\beta \mathrm{H} 1$ cells and human islets ${ }^{8}$ using the RNeasy Mini Kit (Qiagen) which retrieves RNA molecules longer than 200 nucleotides, as previously described in detail ${ }^{58}$. RNA integrity number (RIN) values were evaluated using the Agilent bioanalyzer 2100 (Agilent Technologies, Wokingham, UK). All the samples had RIN values of $>8$ (Supplementary Note 8 ).

RNA-seq libraries were sequenced on a HiSeq 2000 plataform to produce 100bp long paired-end reads with an average of 180 million reads per replicate (EndoC- $\beta \mathrm{H} 1 \mathrm{n}=5$ ). Reads were aligned using TopHat (version 2.0.13) ${ }^{59}$ to GChr37 genome with default parameters. Afterwards, reads were assigned to Gencode version 18 gene annotation ${ }^{60}$ using htseq-count (version 0.6.1p1) ${ }^{61}$ with default parameters. The RNA-seq of 5 human islet preparations $^{8}$ was used for comparison and processed in an identical way.

\section{Differential analysis ATAC-seq, ChIP-seq and RNA-seq}

For both ATAC-seq and ChIP-seq, aligned reads from all replicates were merged into a single BAM file to identify a comprehensive set of peaks. We next used such peak set to compute read counts, separately for each replicate and condition. In the case of RNA-seq data, the output of htseq-count ${ }^{61}$ was used as the input matrix for downstream analysis. The generated matrices were normalized and differential analysis was performed using DESeq $2^{62}$ using a paired sample design (Supplementary Note 9). Thresholds for significance were set at an FDR adjusted $P<0.05$ and $\mid \log _{2} \mathrm{FCl}>1$. All regions/genes that did not reach significance or did not pass $\log _{2}$ fold change cutoff were classified as stable/equalregulated. 


\section{Proteomics}

For the proteomic analysis 1.5 million EndoC- $\beta \mathrm{H} 1$ cells treated or not with cytokines (IL-1 $\beta$ + IFN- $\gamma$ ) were processed using the Metabolite, Protein and Lipid Extraction (MPLEx) approach $^{63}$ (Supplementary Note 10).

Collected data were processed using Decon2LS_V2 ${ }^{64}$ and DTARefinery ${ }^{65}$, both using default parameters, to recalibrate the runs and generate peak lists. Peptide identifications were done using $\mathrm{MSGF}^{66}$ by searching peak lists against islet protein sequences deduced from a transcriptomics experiment ${ }^{47}$ and supplemented with keratin sequences $(32,780$ total protein sequences) (Supplementary Note 10).

Extracted reporter ion intensities (Supplementary Note 10) were then converted into $\log _{2}$ and normalized by standard median centering. Proteins were quantified using a Bayesian proteoform discovery methodology (Bayesian proteoform quantification) in combination with standard reference-based median quantification ${ }^{67}$ and were considered significant at a cutoff of $\mathrm{p} \leq 0.05$ based on a paired T-test.

Protein-protein interaction (PPI) network analysis was performed with GeNets ${ }^{68}$ using Metanetworks v1.0 that integrates PPI from InWeb3 ${ }^{69}$ and ConsensusPathDB ${ }^{70}$. Default parameters were applied and Molecular Signatures Database (MSigDB; http:// software.broadinstitute.org/gsea/msigdb/) ${ }^{71}$ enriched pathways were overlaid.

\section{Defining classes of induced regulatory elements}

In order to characterize chromatin accessibility dynamics upon exposure of human islets and EndoC- $\beta \mathrm{H} 1$ to pro-inflammatory cytokines, we processed the results obtained from the DESeq2 differential analysis and computed the overlap between ATAC-seq peaks and H3K27ac enriched sites, allowing a 200bp gap. Regions annotated as "stable" for both ATAC-seq and H3K27ac assays were classified as Stable Regulatory Elements (SREs). Regions classified as either "stable" or "gained" in ATAC-seq differential analysis and as "gained" in H3K27ac were classified as Induced Regulatory Elements (IREs).

Induced Regulatory Elements (IREs) were classified in two groups: "Opening” IREs $(\mathrm{n}=2,436)$, corresponding to regions annotated as "gained" for both ATAC-seq and H3K27ac and "primed" IREs $(\mathrm{n}=1,362)$ for regions annotated as "stable" for ATAC-seq and "gained" for H3K27ac. Since opening IREs include a gradient of cytokine-induced chromatin accessibility changes, we next selected only those opening regions that were completely closed prior to the cytokine exposure. For this purpose, we considered newly open chromatin those opening ATAC-seq peaks that were not called in the control samples using a relaxed threshold $(P \unlhd) .05)$. Such analysis allowed us to identify a subset of 1,716 opening regions that we named "neo" IREs. A similar approach was used to identify macrophage latent enhancers ${ }^{7}$.

See Supplementary Note 11 for sequence conservation analysis performed at the different classes of induced regulatory elements. 


\section{Assignment of regulatory elements to target genes}

In order to annotate regulatory elements as distal or proximal, we assigned each regulatory element to the nearest TSS of a coding gene (using gencode v18 annotation ${ }^{60}$ ). Those regions that lie within $2 \mathrm{kbs}$ from the nearest TSS were annotated as promoters while the rest were considered as distal regulatory elements.

To test association between different classes of open chromatin and changes in gene expression and protein abundance (Fig. 1d-e, Extended Data Fig. 2e, 3a) in an unbiased manner, we assigned ATAC-seq sites to genes closer than $15 \mathrm{~kb}$ from its TSS. For analyzing the additive effect of IREs on gene expression changes, we associated to a gene all IREs within $40 \mathrm{~kb}$ of their TSS (Extended Data Fig. 2f)

Finally, in order to detect all possible IRE gene targets, we assigned to each IRE all upregulated genes whose TSS was closer than $40 \mathrm{~Kb}$. When an up-regulated gene could not be found in $<40 \mathrm{~Kb}$, the IRE was assigned to the closest, but $<1 \mathrm{Mb}$ far, induced gene (Extended Data Fig. 4a and Supplementary Table 1-2. See Supplementary Note 12).

\section{Sequence composition and transcription factor analysis}

De novo motif analysis was performed using HOMER (version 4.8.2) ${ }^{72}$ findMotifGenome.pl tool with parameters "-size given -bits -mask". Only enriched sequences present in more than $1.5 \%$ of targets were retained. Selection of best matches was performed as follows: all matches with scores over 0.80 were included in the table. For those hits without any match over 0.80 , the top 3 hits were selected and their score was included in the table (Extended Data Fig. $1 \mathrm{~g}$ and 3 e,f).

To assay motif colocalization, we used all motifs instances identified in the de novo analysis in primed enhancers. First we used the findMotifGenome.pl tool from HOMER to map all these motif instances in primed enhancers and SRE enhancers (i.e. excluding all sites $<2 \mathrm{~Kb}$ from a TSS). Next, the motif co-localization was calculated by counting motif pairs found in each ATAC-seq peak. Significance was determined by Fisher Exact test comparing colocalization of motifs pairs in IREs vs. SREs distal regions. Only significant pairs (Fisher Exact test, FDR adjusted $P<0.001$ ) were retained (Extended Data Fig. 3 g,h).

To evaluate islet-specific transcription factors occupancy, we used ChIP-seq bam files for PDX1, NKX2.2, FOXA2, NKX6.1 and MAFB ${ }^{9}$. We computed the read coverage in the regions of interest over 10bp bins. Reads were quantile-normalized, mean counts in each bin for each transcription factor were calculated and the mean for all TFs was plotted (Figure $2 d)$.

To identify footprints from the ATAC-seq data, we generated tag directories with all ATACseq replicates in each condition using HOMER makeTagDirectory. Neo and primed enhancers were centered on the ISRE motif matrix annotated with annotatePeaks.pl with options "-center motif1.motif -size given" and tag means for 5' and 3' read ends were obtained using annotatePeaks.pl with options "-size -100,100 -hist 1 -d tagsDir". The resulting 5 ' ends were plotted using ggplot ${ }^{73}$ (Figure 2e). 
In order to create a non-redundant dataset of motifs for the gene regulatory network analysis (Extended Data Fig. 4a), motifs from primed and opening enhancers were reduced to a nonredundant set with the compareMotifs.pl script from HOMER using a similarity score of 0.7 as threshold for merging similar motifs. The motifs were then mapped to primed and opening enhancers using annotatePeaks.pl.

\section{K Infinium MethylationEPIC Array}

DNA from EndoC- $\beta \mathrm{H} 1$ cells exposed or not to IL- $1 \beta$ and IFN- $\gamma$ for $48 \mathrm{~h}$ as described above (5 replicates per condition) was extracted using QIAamp DNA Mini kit (Qiagen, Venlo, The Netherlands). $1 \mu \mathrm{g}$ DNA aliquots $(\mathrm{n}=10$ ) were processed for $850 \mathrm{~K}$ Infinium MethylationEPIC Array (Illumina) as previously described ${ }^{74}$.

The resulting array signals were processed and analyzed using RnBeads R package ${ }^{75}$. The method used by RnBeads for assessing differences between groups consists on fitting a hierarchal linear model (empirical Bayes method from the limma package ${ }^{76}$ ) using M-values ( $\log$ of $\beta$-values) as metrics to measure methylation levels ${ }^{77}$. All $P$-values were corrected for multiple testing using the Benjamini-Hochberg method for controlling the False Discovery Rate (FDR). CpGs were considered as differentially methylated when FDR adjusted $P<0.05$ and absolute difference in methylation $\beta$-values between cytokine and control samples was $>0.2$ ( $20 \%$ changes in methylation). Information on the differentially methylated CpGs can be found in Supplementary Table 7.

\section{UMI-4C}

UMI-4C was performed as described ${ }^{25}$ with minor modifications (Supplementary Note 13). To increase molecular complexity, each library was obtained by pooling 5-10 PCRs per viewpoint. The PCR primers used in UMI-4C are listed in Supplementary Table 8. Each library was sequenced to a depth of $>1 \mathrm{M} 75 \mathrm{bp}$ long paired-end reads using either NextSeq or HiSeq 2500 platforms.

Paired-end reads were demultiplexed according to the viewpoint sequence using fastx-multx from ea-utils ${ }^{78}$ and analyzed using umi4cPackage ${ }^{25} .4 \mathrm{C}$ tracks were created by selecting viewpoint-specific reads, aligning them to the genome and extracting the number of UMIs using the $p 4 c$ reate 4 CseqTrack function (see quality control statistics in Supplementary Table 9). Cytokine-treated profiles were then scaled to the control profile using the umi 4 cPackage package function $p 4 c$ SmoothedTrendComp. Profiles were also smoothened based on the total number of UMIs present in a $2 \mathrm{Mb}$ region centered on the viewpoint and excluding $3 \mathrm{~kb}$ around it. The following formula was used to calculate the minimum UMIs needed for smoothing. If the fragment did not reach this minimum, it was then merged with successive fragments until minimum was reached.

$$
\text { Minimum UMIs }=\frac{\sum \text { UMIs region }}{2000} \times 50
$$

In order to detect differential chromatin contacts we focused on a $2 \mathrm{Mb}$ region centered on the viewpoint, but excluding $1.5 \mathrm{~kb}$ on each side of the viewpoint. We then partitioned the 
region into windows of width proportional to the mean restriction fragment length in the region $\left(\right.$ mean $\left._{\text {fragment }}\right)$ :

$$
\text { width }_{\text {window }}=\text { mean }_{\text {fragment }} \times 20
$$

Differential contacts analysis was performed for each of the above-defined windows using a Chi-squared test, comparing UMIs in such windows with the total number of UMIs in the $2 \mathrm{Mb}$ region. Windows with a Chi-squared $P<0.05$ were highlighted in Fig 3a,b and Extended Data Fig 5b-d by a small black diamond. To quantify the chromatin contact changes, we counted the number of cytokine-treated and control UMIs for each window and computed their odds ratio based on the total UMI counts in the region, following the formula

$$
\mathrm{OR}_{\text {window }}=\frac{\mathrm{CTRL}_{\text {region }} \times \mathrm{CYT}_{\text {window }}}{\mathrm{CTRL}_{\text {window }} \times \mathrm{CYT}_{\text {region }}}
$$

where CTRL and CYT represent the number of UMIs in control (CTRL) and cytokineexposed (CYT) conditions.

\section{Variant enrichment analyses}

We used the Variant Set Enrichment Analysis (VSE) R package ${ }^{79}$ to assess the enrichment of T1D and T2D risk variant for IRE and SRE regulatory annotations. We first selected from the NHGRI-EBI GWAS Catalog ${ }^{26}$ all "leading" SNPs with disease trait matching either "Type 1 diabetes" or "Type 2 diabetes" (date 2019-04-24). Next, we extended our collection of associated variants to all those in strong LD ( $\mathrm{R}^{2} \searrow 0.8$, EUR) with the lead SNP (source of LD information 1000 Genomes Project phase $3^{80}$ ). These SNPs and their proxies were used to generate the Associated Variant Set (AVS) ${ }^{79}$, resulting in 83 disjointed regions for T1D and 389 for T2D, after removing shared loci between T1D and T2D. A null distribution or Matched Random Variant Set (MRVS), matched in size and structure to the original AVS, was generated from 1000 Genomes Project phase 3 by permutating the AVS 500 times. The number of independent SNPs from the AVS overlapping the regulatory annotations was computed and compared with the intersections obtained with the MRVS. The enrichment score was defined as the number of standard deviations that the overlapping tally deviates from the null overlapping tally median. The exact $P$-value was then calculated by fitting a density function into the null distribution derived from the MRVS. This $P$-value was finally corrected for multiple-testing using the Bonferroni method. Enrichments or depletions with a Bonferroni adjusted $P<0.05$ were considered statistically significant (Fig. 4a and Extended Data Fig. 6a).

T1D associated regions were generated by selecting all SNPs in strong $L D\left(\mathrm{R}^{2} \searrow 0.8, \mathrm{EUR}\right)$ with the T1D leading SNPs. We defined the risk loci boundaries using the most upstream and the most downstream SNPs. We next merged the overlapping loci to obtain a total of 71 T1D risk regions. All T1D associated regions containing IREs and T1D risk variants directly overlapping human islet cytokine-induced regulatory elements are shown in Supplementary Table 3. For this analysis, in order to extract all possible cytokine induced regulatory 
element located at T1D risk loci we used a less stringent set of human islet IREs by lowering the H3K27ac $\log _{2}$ fold-change threshold from 1 to 0.8 .

For details regarding the GWAS association analysis see Supplementary Note 14.

\section{Luciferase reporter assays}

For episomal reporter assays in the EndoC- $\beta \mathrm{H} 1$ cell line, selected human cytokine-induced regulatory elements regions were first amplified from genomic DNA with primers (Supplementary Table 10) containing XhoI/HindIII restriction sites. The amplicons were next cloned into the PGL4.23[luc2/minP] Luciferase Reporter Vector (Promega) as previously described ${ }^{81}$. Briefly, the amplicon and the vector were simultaneously digested. Next, the vector was dephosphorylated with FastAP (Thermo Scientific). The DNA was then purified and ligated with a T4 DNA ligase (Promega). Next, the generated Reporter Vectors were transformed into E. coli (DH5a) and purified using the Nucleospin Plasmid (740588.250, MN, Düren, Germany).

Site-directed mutagenesis was used to introduce single nucleotide variants into the generated construct. The variants were generated by PCR using the primers shown in Supplementary Table 10. The parental supercoiled double-stranded DNA was digested with DpnI (NEB, 174R0176S) $1 \mathrm{~h}$ at $37^{\circ} \mathrm{C}$ and the constructs were transformed in competent E. coli cells (DH5a) by thermal shock. Finally, the introduced variants were checked by Sanger sequencing.

EndoC- $\beta \mathrm{H} 1$ cells were transfected in 24 well plates, at a density of 300,000 cells per well, with $200 \mathrm{ng}$ of reporter vectors or empty vectors, plus $20 \mathrm{ng}$ of phRL-CMV Renillaluciferase to control for transfection efficiency.

Transfections were performed with lipofectamine 2000 (Invitrogen) for 8h, according to manufacturer instructions. Upon transfection EndoC- $\beta \mathrm{H} 1$ medium was supplemented with $2 \% \mathrm{FBS}^{82}$ and exposed or not to the cytokines for $48 \mathrm{~h}$. After $48 \mathrm{~h}$, the cells were assayed using the Dual Luciferase Assay (Promega, Madison, USA), following manufacturer instructions. The luciferase units were measured using VICTOR Multilabel Plate Reader (PerkinElmer). Firefly luciferase activity was normalized to Renilla luciferase activity and then divided by values obtained for the empty pGL4.23. The assays were performed in at least three independent experiments.

Statistical differences were calculated using a one-way ANOVA test. P-values were then corrected with Bonferroni correction.

\section{Reporting Summary:}

Further information on research design is available in the Life Science Reporting Summary linked to this article.

\section{Data availability:}

Datasets for induced regulatory elements (IREs) are available for download and visualization at the Islet Regulome Browser ${ }^{83}$ (www.isletregulome.com). 
Raw sequencing reads for the different high-throughput assays can be accessed at GEO with the following identifiers: GSE123404 (ATAC-seq), GSE133135 (ChIP-seq H3K27ac), GSE137136 (RNA-seq) and GSE136865 (UMI-4C). Raw proteomics data can be accessed at ProteomeXchange with the identifier PXD011902.

Code availability:

Code and scripts used in this paper are available upon request.

\section{Extended Data}



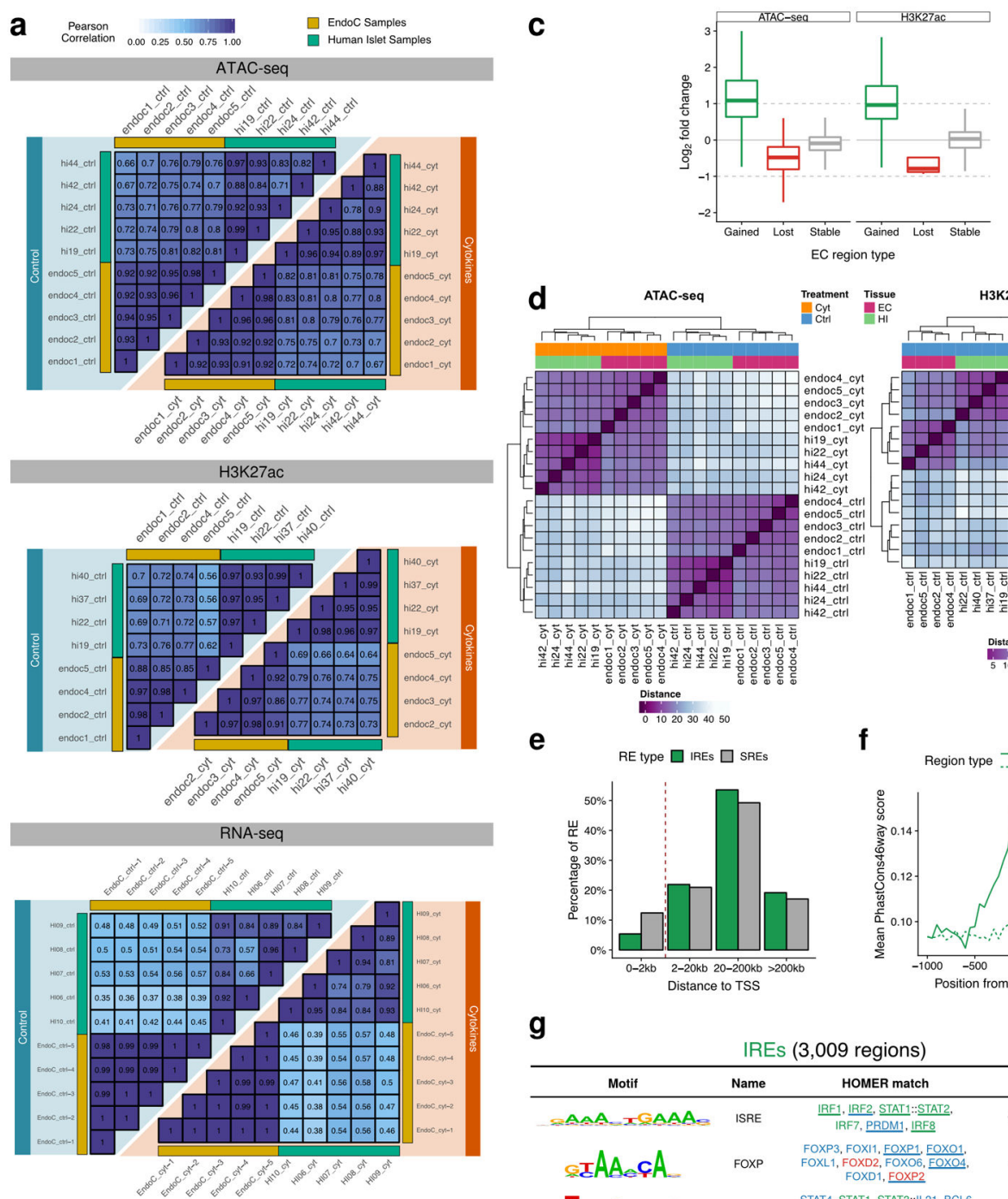

e
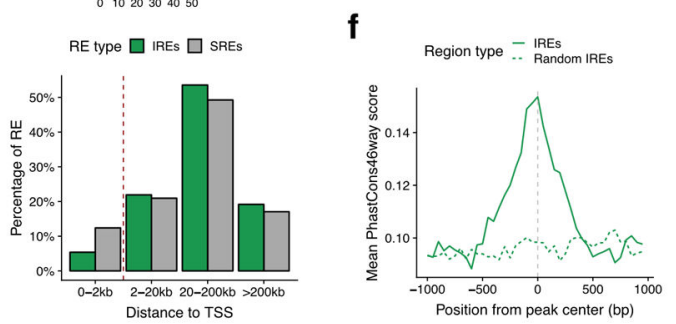

g
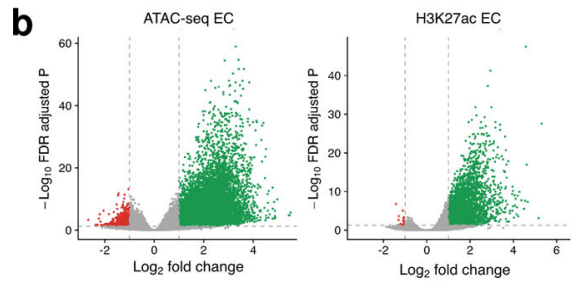

\begin{tabular}{|c|c|c|c|c|}
\hline \multicolumn{5}{|c|}{ IREs (3,009 regions) } \\
\hline Motif & Name & HOMER match & $P$ & \% targe \\
\hline$\triangle A A_{-} X \triangle A A A=$ & ISRE & 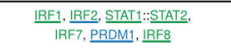 & $1 e^{1356}$ & 51.71 \\
\hline$\triangle A A_{\&} c A$ & Foxp & 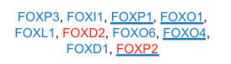 & $1 e^{30}$ & 44.1. \\
\hline $\mathrm{T}_{\mathrm{I}=\mathrm{C}}$ & STAT & $\begin{array}{l}\text { STAT4, STAT1, STAT3:L21, BCL6, } \\
\text { STATSA:STATEB }\end{array}$ & $1 e^{31}$ & 15.29 \\
\hline$T_{\triangle} A_{\pi} A_{-T_{\Theta \Theta}}$ & HNF 1 & HNF 18, ,HNF1A & $1 e^{200}$ & 4.62 \\
\hline $\mathrm{CCA}_{\mathbf{T}} \mathrm{CT} \mathrm{G}_{\mathrm{I}}$ & $\begin{array}{c}\text { BHLH } \\
\text { (NEUROODI) }\end{array}$ & 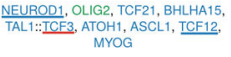 & ${ }^{1 e^{25}}$ & 29.88 \\
\hline $\mathrm{GG}_{\triangle A A} \mathrm{CCC}$ & NFKB & RELA, NFEB & $1 e^{21}$ & 5.85 \\
\hline$I_{A C T} C_{A C}$ & DZIP (AP1) & 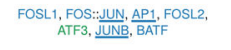 & $e^{1 / 8}$ & 17.35 \\
\hline $\mathrm{T} \in C T_{\triangle A} A \mathrm{~A}$ & MAF & $\begin{array}{l}\text { NRL, TBX20, MAFE, MAFA, } \\
\text { MAFF, MAFE }\end{array}$ & $e^{1 / 8}$ & 16.38 \\
\hline$C A A A C C_{\triangle}$ & IRF & IRFA (0.76), BuNXX (0.09) & $1 e^{13}$ & 11.67 \\
\hline
\end{tabular}

Extended Data Fig. 1. Chromatin characterization of human pancreatic $\beta$ cells exposed to proinflammatory cytokines.

a, Pearson correlation values between replicates in different assays and conditions (see Supplementary Note 2). b, Volcano plots of ATAC-seq (left) and H3K27ac ChIP-seq (right) changes obtained after exposure of EndoC- $\beta \mathrm{H} 1$ to IFN- $\gamma$ and IL-1 $\beta$; green and red dots correspond to sites with $\log _{2} \mathrm{FCl}>1$ and FDR adjusted $P<0.05$ as calculated by fitting a negative binomial model in DESeq2. Chromatin changes are classified as "gained" and "lost" chromatin sites whereas non-significant changes are defined as "stable". c, Chromatin accessibility and H3K27ac enrichment changes observed in EndoC- $\beta \mathrm{H} 1$ are largely 
replicated in human pancreatic islets as illustrated by the distribution of $\log _{2}$ fold change at regions as classified in $\mathbf{b}$ in EndoC- $\beta \mathrm{H} 1$. Dotted lines indicate $\log _{2}$ fold change thresholds $\left(\log _{2} \mathrm{FC} \mid>1\right)$. Box plot limits show upper and lower quartiles, whiskers extend to 1.5 times the interquartile range and the notch represents the confidence interval around the median. $\mathbf{d}$, Hierarchical clustering using normalized ATAC-seq and H3K27ac ChIP-seq read counts at EndoC- $\beta$ H1 IREs shows that samples cluster according to treatment, suggesting that the differences caused by the proinflammatory cytokines are greater than those derived by the sample heterogeneity. HI=Human pancreatic islets, EndoC=EndoC- $\beta \mathrm{H} 1 \mathbf{e}$, Distribution of distances to nearest TSS for the different types of regulatory elements, showing that IREs, compared with stable regulatory elements (SREs), are preferentially located distally to TSS. f, Mean sequence conservation score of IREs and a randomized set of IREs in placental mammals. Peaks were extended from the center $1 \mathrm{~kb}$ to each direction and mean score was calculated in 50bp windows. g, Sequence composition analysis of IREs $(n=3,009)$ illustrating the top identified de novo motifs. Colors for matched genes correspond to RNAseq (name) or protein (underlined) status (red=down-regulated, blue=equal-regulated, green=up-regulated, black/no line $=$ not expressed/detected). 


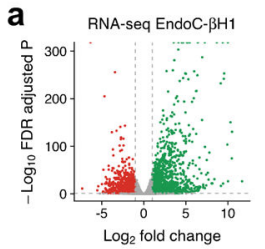

\section{d}

b
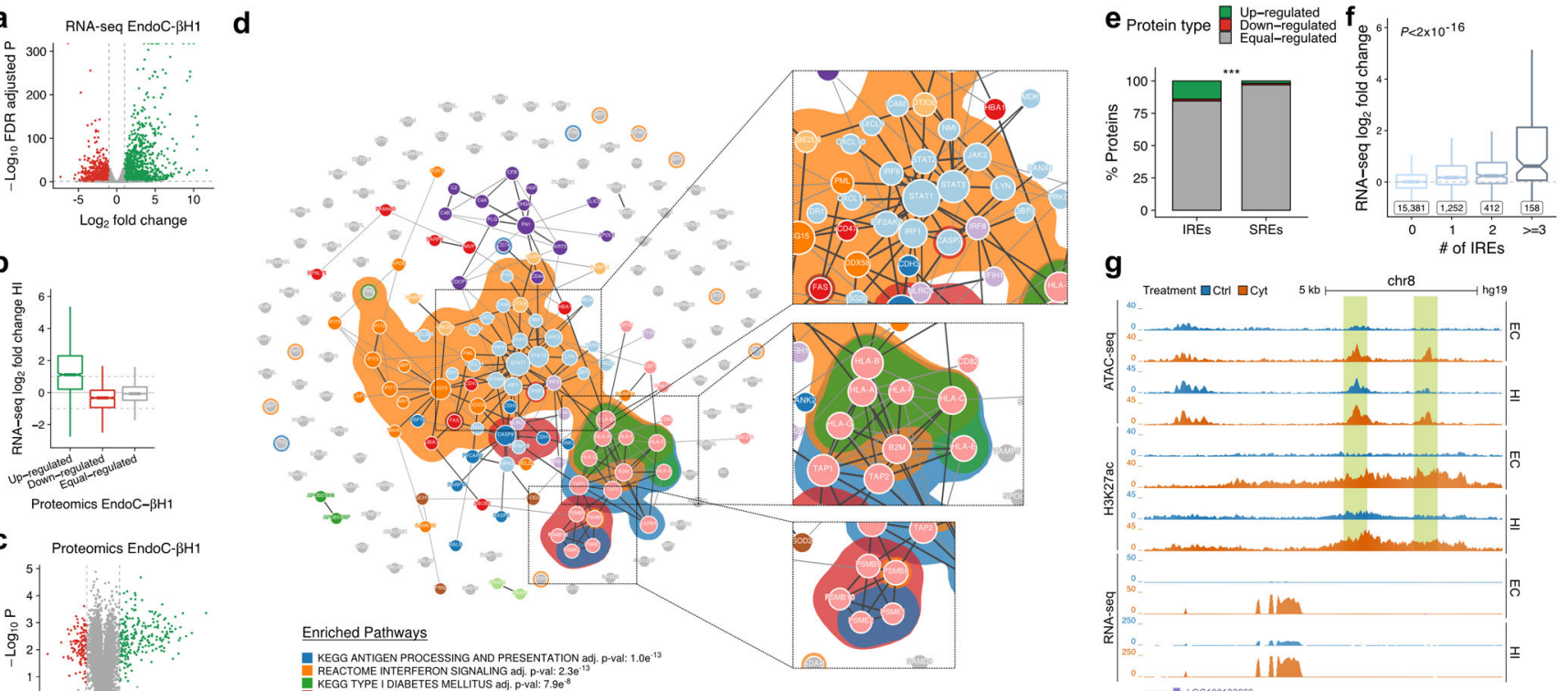

C Proteomics EndoC- $-\mathrm{BH} 1$

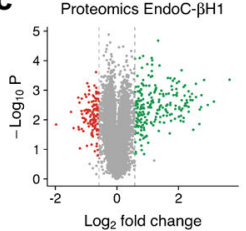
KEGG ANTIGEN PROCESSING AND PRESENTATION ad, p.val: 1.0e

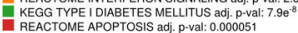

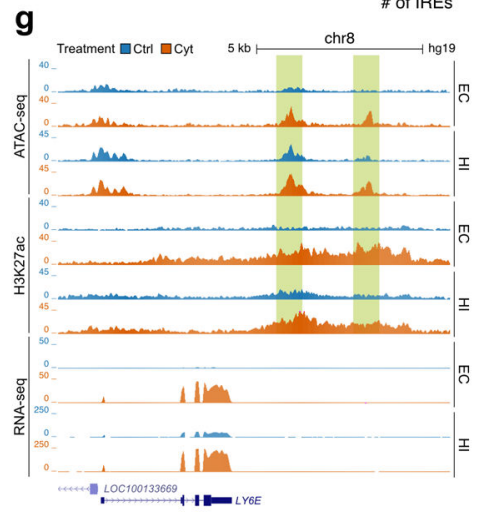

Extended Data Fig. 2. Exposure to pro-inflammatory cytokines drives changes in the transcriptome and proteome of pancreatic $\beta$ cells.

a, Volcano plot of RNA-seq genes, showing up-regulated genes (green) and down-regulated genes (red) upon exposure of EndoC- $\beta \mathrm{H} 1$ to cytokines. Vertical lines indicate the $\log _{2}$ fold change threshold ( $\left.\mid \log _{2} \mathrm{FCl}>1\right)$ and horizontal line indicates the FDR adjusted $P$ cutoff for significance (FDR adjusted $P<0.05$ ) calculated by fitting a negative binomial model in DESeq2. b, Distribution of RNA-seq counts in human islet samples in the genes previously classified as up, down or equal-regulated in EndoC- $\beta \mathrm{H} 1$ cells. Boxplot limits show upper and lower quartiles, whiskers extend to 1.5 times the interquartile range and the notch represents the confidence interval around the median. c, Volcano plot for multiplex proteomics, showing in green the up-regulated proteins and in red the down-regulated, which have a Q-value $<0.1$ and $\log _{2} \mathrm{FCl}>0.58$. Vertical lines indicate the $\log _{2}$ fold change thresholds. d, Protein-protein Interaction (PPI) network generated from up-regulated proteins after cytokine exposure. Node color indicates belonging to same interacting community and background corresponds to specific pathway enrichment. e, Proportion of up, equal or down-regulated proteins encoded by genes located $<15 \mathrm{~kb}$ from IREs or SREs. $* * *$ Chi-squared test $P<0.001$. f, An additive effect on gene up-regulation was observed for multiple IREs located at $<40 \mathrm{~kb}$ of a gene. Box plot limits show upper and lower quartiles, whiskers extend to 1.5 times the interquartile range and the notch represents the confidence interval around the median. ANOVA $P<2.2 \times 10^{-16}$. g, View of the LY6Elocus, whose expression is induced after cytokine exposure and is coupled with chromatin changes in the vicinity. 

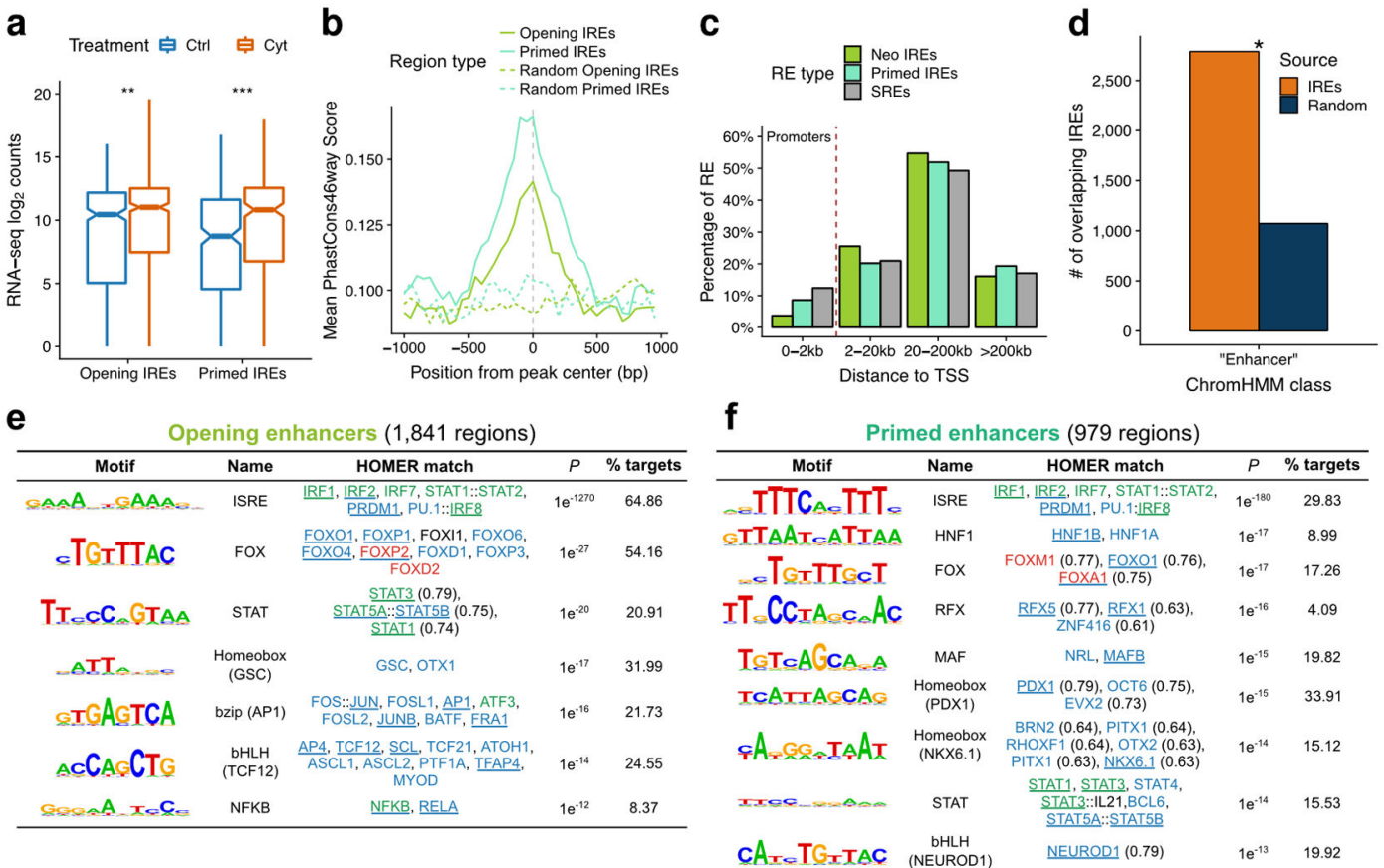

g
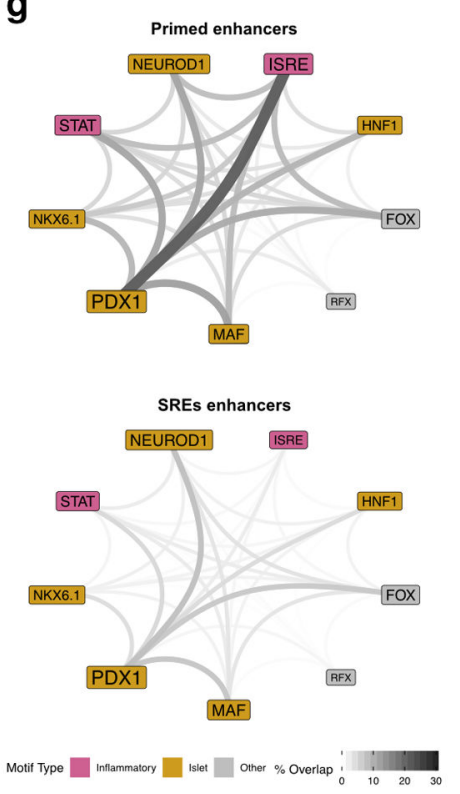

h

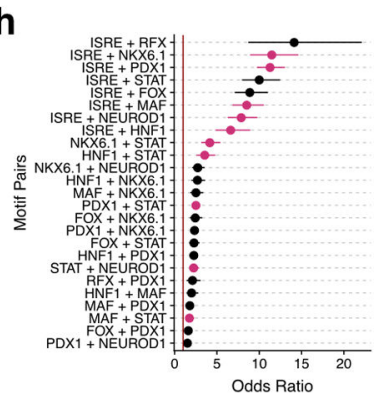

j

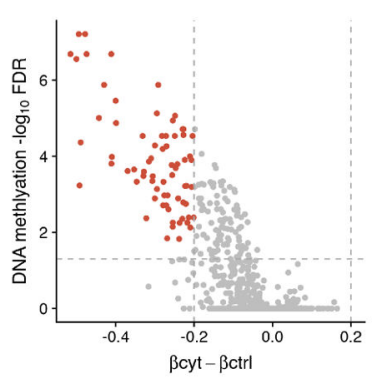

i
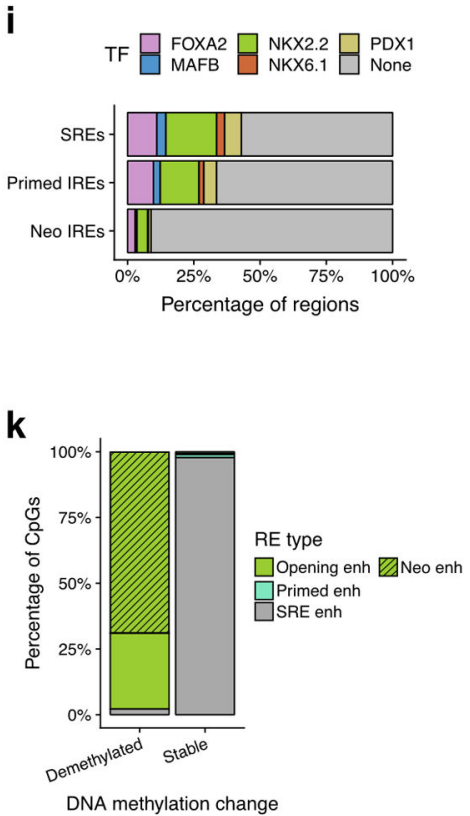

Extended Data Fig. 3. Characterization of $\beta$-cell IREs.

a, Genes associated to different classes of IREs (classified as in Fig. 2a) show cytokineinduced expression in EndoC- $\beta$ H1. CYT=cytokine exposed, CTRL=control. Boxplot limits show upper and lower quartiles, whiskers extend to 1.5 times the interquartile range and notch represents the median confidence interval. ***Wilcoxon test $P<0.001$. b, Sequence conservation score of IREs and a corresponding randomized set used as control. c,

Distribution of distances to nearest TSS of the different classes of open chromatin sites. Line indicates the threshold used to classify them as "promoters". d, Number of IREs overlapping regions annotated as "Strong" or "Weak" enhancers by ENCODE ChromHMM. *Chi- 
squared $P<2 \times 10^{-16}$. e, f, Top hits for de novo motif analysis in opening (e) and primed enhancers (f). Colors for matched genes correspond to RNA-seq (name) or protein (underlined) status (red=down-regulated, blue=equal-regulated, green=up-regulated, black/no-line=not-expressed/detected). $\mathbf{g}$, Diagram showing the percentage of colocalization between the TF binding sites identified by de novo motif analysis in SRE and primed enhancers (i.e. excluding sites $<2 \mathrm{~Kb}$ from a TSS). Label size indicates number of regions containing the TF binding sites and line width/intensity percentage of regions in which two motifs colocalize. $\mathbf{h}$, Odds-ratio for finding a motif pair in the same enhancer in primed vs. SRE. Only significant pairs (FDR-adjusted Fisher Exact test $P<0.001$ ) are shown. Immune and islet-specific TF motifs colocalize more often in primed compared to SRE chromatin sites. i, Percentage of overlap between EndoC- $\beta \mathrm{H} 1$ different classes of open chromatin and islet-specific TFs obtained by ChIP-seq in untreated human islets. $\mathbf{j}$, Volcano plot showing differentially methylated sites (depicted in red) in EndoC- $\beta \mathrm{H} 1$ exposed or not to cytokines. Dotted lines indicate the threshold for methylation differences or significance using limma moderated t-test. $\mathbf{k}$, Distribution of demethylated and stable CpGs according to different classes of open chromatin. 


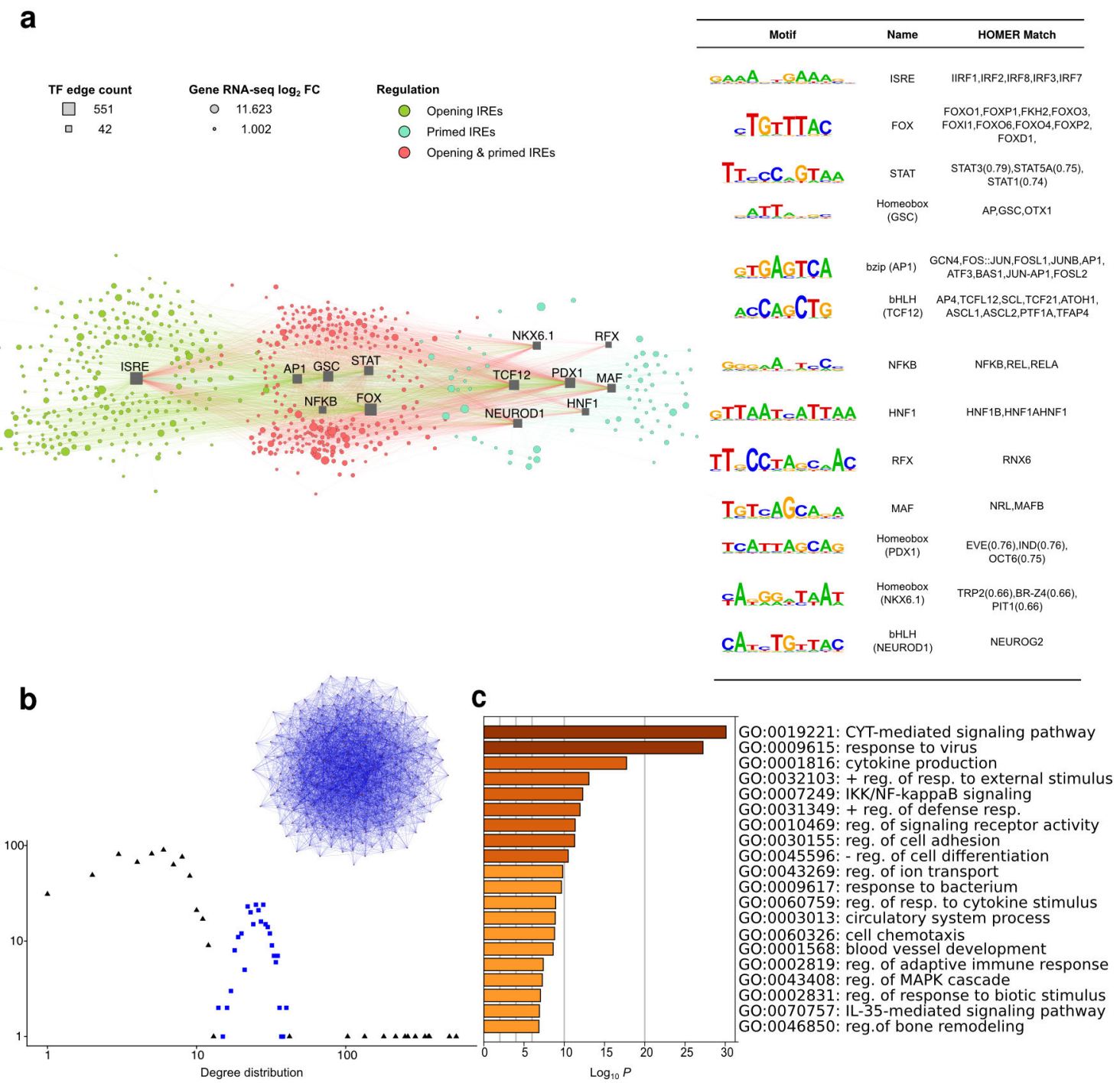

Extended Data Fig. 4. Deconstructing cytokine induced cis-regulatory networks in $\boldsymbol{\beta}$ cells. a, Gene Regulatory Network (GRN) derived from IREs and their putative target genes. Squares represent the IREs inferred TF binding sites (motifs logos and TF matches are shown on the right side) and the ellipses represent their putative target genes (see methods). The size of the squares reflects the number of connections (edge count) while the gene node size reflects the $\log _{2}$ fold change of RNA expression after cytokine exposure. The resulting GRN is an interconnected scale-free network composed of 648 nodes and 3,589 edges.

Genes regulated exclusively by primed IREs are represented in blue while green depicts opening IREs regulated genes. Red denotes genes regulated by both types of IREs. In each of these three groups the representation of the hierarchy is based on the principle of network centrality where authoritative nodes are located more proximal to the core. $\mathbf{b}$, Comparison between the degree distribution of the observed GRN (black triangles) and a random generated network (blue squares) having the same number of nodes and edges. The bellshaped degree distribution of random graph denotes a statistically homogeneity in the degree of small and large nodes. In contrast, the observed network showed a high frequency of 
small degree nodes and a low frequency of highly connected nodes as is typical of a scalefree network. c, Bar plot of gene ontology biological process enrichment analysis. Geneontology analysis was performed using all target genes in the GRN. Functional enrichment analysis was performed by Metascape (http://metascape.org). Only terms with $P<0.001$ and with at least 3 enriched genes were considered as significant. Color is proportional to their $P$ values. 
$a$

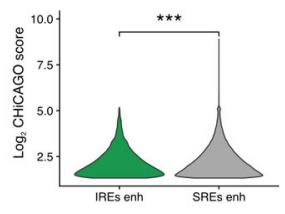

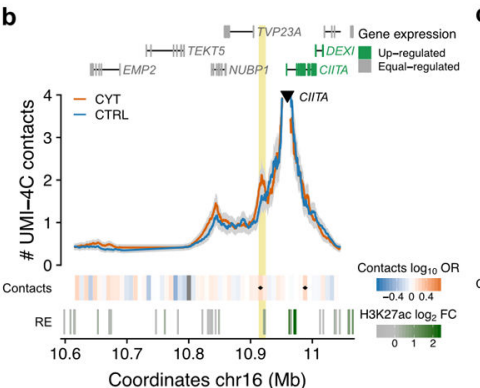

c

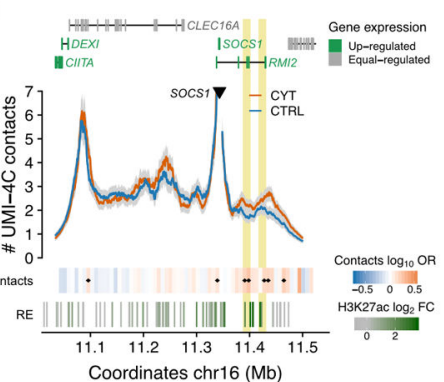

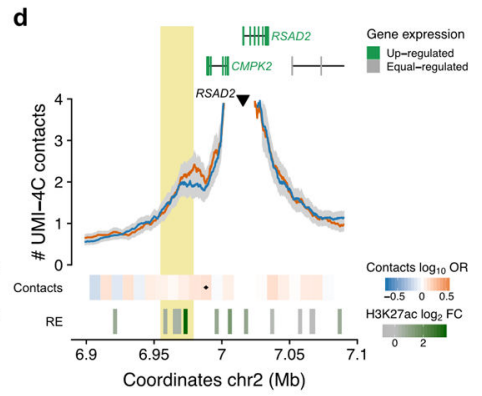

Extended Data Fig. 5. 3D chromatin changes induced by exposure of human islets to proinflammatory cytokines.

a, Violin plots showing the distribution of CHiCAGO scores of contacts, detected by pc-HiC experiments in untreated human islets ${ }^{2}$, between stable and induced enhancers and their target genes. SREs engage chromatin contacts with higher interaction scores compared to those detected for IREs. *** Wilcoxon test $P<0.001$. b, c, d, Views of the 3D chromatin contacts of CIITA (b), SOCS1 (c) and RSAD2 (d) promoters obtained by UMI-4C performed in islets exposed or not to pro-inflammatory cytokines. In yellow we highlight those IREs that gain contacts with the up-regulated gene promoter. A heatmap under the 4C track represents the $\log _{10}$ odds ratio (OR) of the UMI-4C contacts difference in cytokine vs. control and a small black diamond on top of the contact heatmap indicates a significant difference in contacts between cytokine-treated and control samples (Chi-squared $P<0.05$ ). ATAC-seq peaks are represented by rectangles, shaded from gray to green proportionally to the cytokine-induced $\mathrm{H} 2 \mathrm{~K} 27 \mathrm{ac} \log _{2}$ fold change observed at that site. 


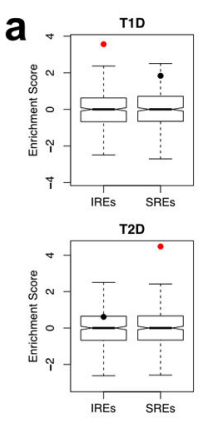

e
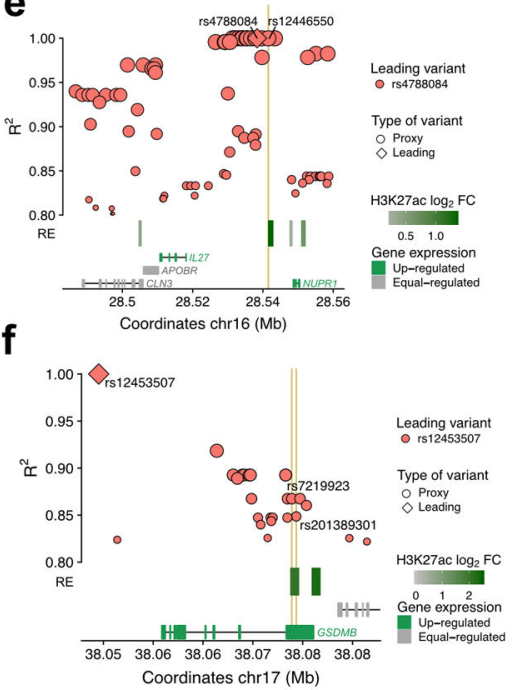

C

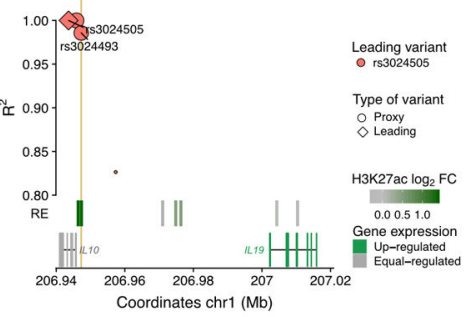

g

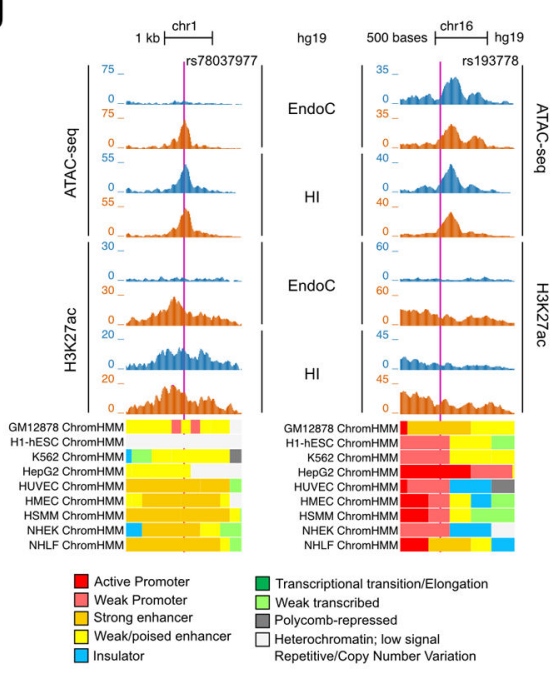

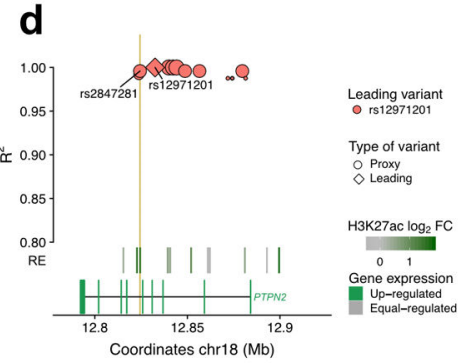

h
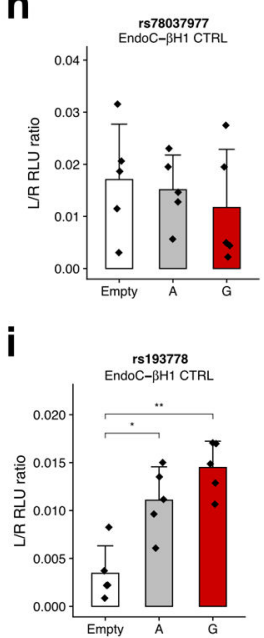

Extended Data Fig. 6. Cytokine-induced islet regulatory elements are enriched in T1D associated variants.

a, EndoC- $\beta$ H1 cytokine-induced regulatory elements (IREs) overlap more often than expected T1D associated variants while the opposite is true for T2D. EndoC- $\beta \mathrm{H} 1$ cytokineinvariant regulatory elements (SREs) are instead enriched for T2D, but not T1D associated variants. Each dot denotes the Varian Set Enrichment (VSE) score in IREs or SREs regions. Boxplot shows the enrichment distribution of the matched null permutated data sets. Red dots indicate that the difference is statistically significant as determined by VSE (Bonferroni adjusted $P<0.05)$. Box plot limits show upper and lower quartiles, whiskers extend to 1.5 times the interquartile range and the notch represents the confidence interval around the median. b-f, Representative regional plots of different T1D risk loci containing T1D variants overlapping IREs and up-regulated genes. $\mathrm{R}^{2}$ values are based on $1 \mathrm{KG}$ phase $3 \mathrm{EUR}$ and the leading SNPs in the locus is represented by a diamond. If different leading variants are present in the same locus, their proxies are depicted in different colors. Yellow squares highlight those variants that overlap a human islet IRE. IREs are depicted as boxes, with the filling color corresponding to the $\mathrm{H} 3 \mathrm{~K} 27 \mathrm{ac} \log _{2}$ fold change. g, The IRE bearing the T1D associated variant rs78037977 is marked by the ENCODE ChromHMM classification as a "strong enhancer" (orange) in other non $\beta$-cell lines (left). ENCODE ChromHMM classification in non $\beta$-cell lines for the IRE bearing the T1D associated variant rs193778. $\mathbf{h}$, i, Allele-specific luciferase experiments for rs78037977 (h) and rs193778 (i) in untreated 
EndoC- $\beta$ H1. ANOVA followed by Bonferroni correction * $P<0.05$; ** $P<0.01$; *** $P<$ 0.001 . Bars represent mean \pm sd. 
a

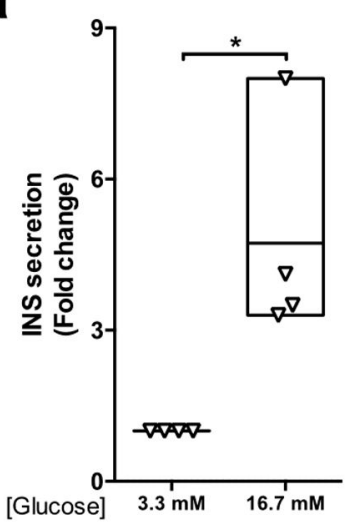

b

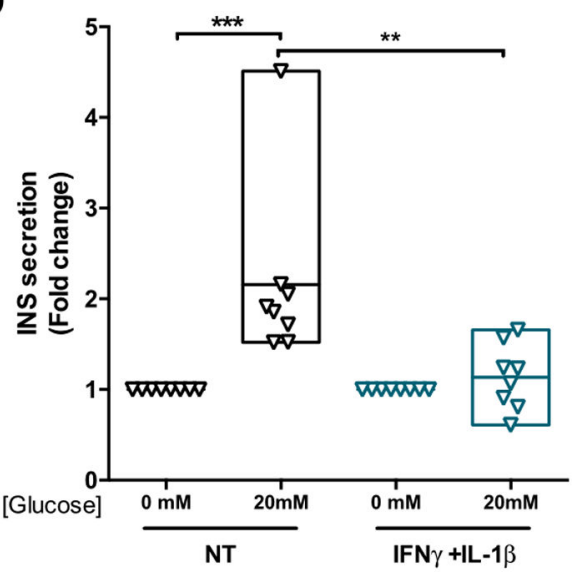

Extended Data Fig. 7. Human islets and EndoC- $\beta$ H1 Glucose-Stimulated Insulin Secretion (GSIS).

GSIS was assessed, in pancreatic human islets (a) and EndoC- $\beta \mathrm{H} 1$ cells (b). In the case of EndoC- $\beta \mathrm{H} 1$ cells, the experiments were performed upon exposure or not to IFN $\gamma(1000$ $\mathrm{U} / \mathrm{ml})+\mathrm{IL} 1 \beta(50 \mathrm{U} / \mathrm{ml})$ for $48 \mathrm{~h}$. Data are mean plus range of four to eight independent experiments, and are expressed as the ratio between glucose stimulated and basal insulin secretion. $* P<0.05, * * P<0.01, * * * P<0.001$, for the indicated comparisons (paired test (a) or ANOVA followed by Bonferroni correction (b)). NT=Non treated. 


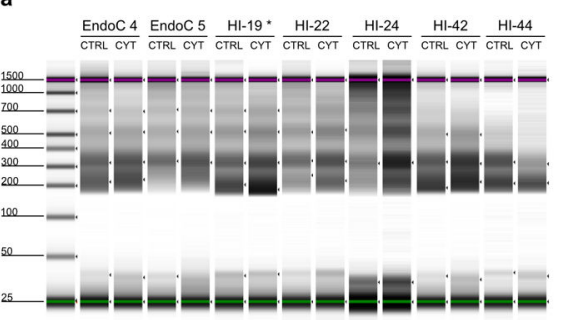

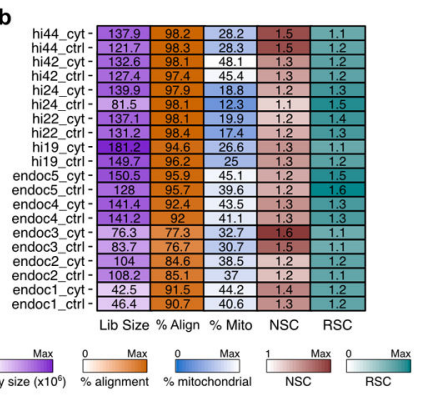

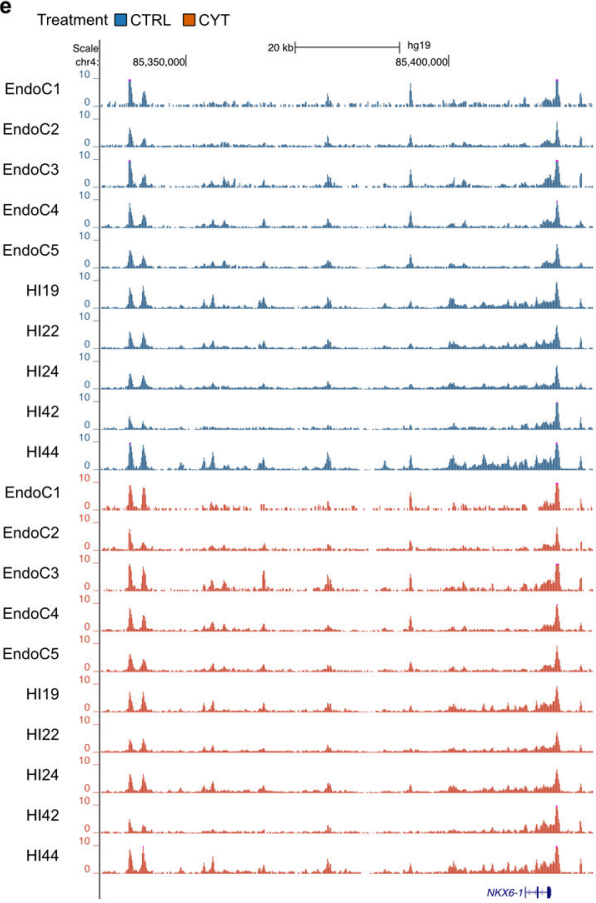

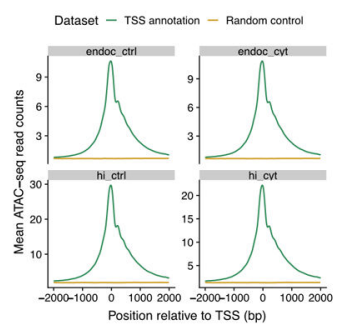

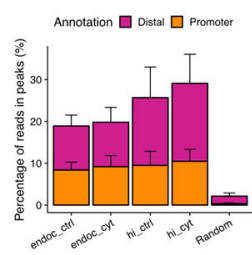

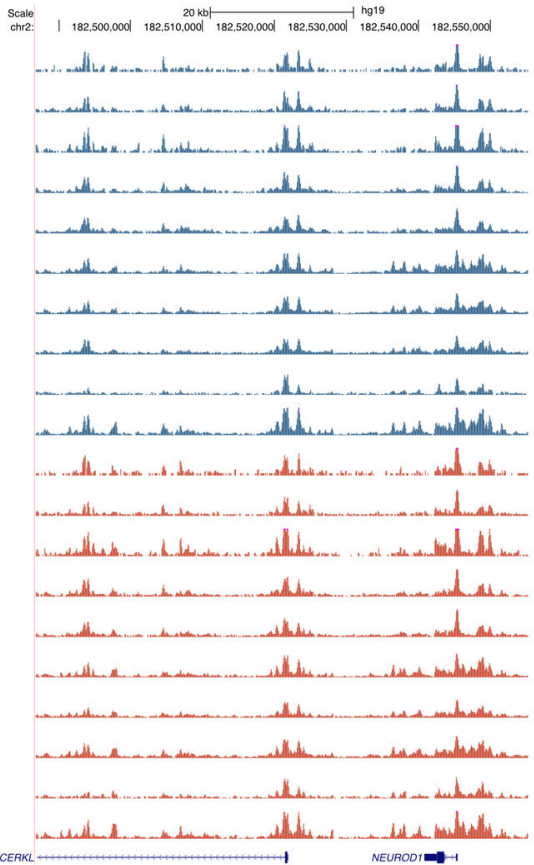

Extended Data Fig. 8. ATAC-seq quality control.

a, Agilent TapeStation profiles obtained by chromatin tagmentation of human islets and EndoC- $\beta \mathrm{H} 1$ samples showing the laddering pattern of ATAC-seq libraries. The band sizes correspond to the expected nucleosomal pattern. *Notice that samples HI-19 CTRL and CYT were used as examples to illustrate the expected fragment distribution pattern in ATAC-seq experiments in Raurell-Vila et al. ${ }^{52}$. b. Summary of per-replicate sequencing metrics, showing total library sizes, percentage of aligned reads, percentage of mitochondrial aligned reads, normalized strand cross-correlation coefficient (NSC, values significantly lower than $1.1(<1.05)$ tend to have low signal to noise or few peaks) and relative strand cross-correlation coefficient (RSC, values significantly lower than $1(<0.8)$ tend to have low signal to noise). c, TSS enrichment over a $4 \mathrm{~kb}$ window centered on genes TSS compared to a set of genes randomized along the genome, showing the expected pattern of open chromatin centered on the TSS. d, Percentage of total reads found at called open chromatin peaks classified as distal ( $>2 \mathrm{~kb}$ from TSS) or promoters ( $\iota \mathrm{kb}$ from TSS) compared to a randomized set of peaks. e, UCSC views at islet-specific loci (NKX6.1, PDX1 and $N E U R O D 1)$ showing the high reproducibility of ATAC-seq profiles among independent replicates. 


\section{Supplementary Material}

Refer to Web version on PubMed Central for supplementary material.

\section{Acknowledgments:}

L.Pasquali was supported by grants from the Spanish Ministry of Economy and Competitiveness (nos. BFU2014-58150-R and SAF2017-86242-R), Marató TV3 (no. 201624.10) and a young investigator award from the Spanish Society of Diabetes (Ayuda SED a Proyectos de Investigación, no. 2017-SED). L.Pasquali is a recipient of a Ramon y Cajal contract from the Spanish Ministry of Economy and Competitiveness (no. RYC-2013-12864). The Pasquali lab is further supported by Instituto de Salud Carlos III (no. PIE16/00011). M.R. is supported by an FI Agència de Gestió d'Ajuts Universitaris i de Recerca PhD fellowship (no. 2019FI_B100203). J.J. was supported by a Marie Skłodowska-Curie Actions fellowship grant from the Horizons 2020 European Union (EU) Programme (project no. 660449). M.I.A. was supported by a FRIA fellowship from the Fonds de la Recherche Scientifique (FNRS) (no. 26410496). Human islets were provided through the European Consortium for Islet Transplantation distribution program for basic research supported by JDRF (no. 31-2008-416). D.L.E. was supported by the Walloon Region through the FRFS-WELBIO Fund for Strategic Fundamental research (no. CR-2015A-06s and CR-2019C-04) and by grants from the Fonds National de la Recherche Scientifique (no. T003613F), the Horizon 2020 Programme (project T2Dsystems, no. GA667191), Brussels Capital Region Innoviris (project DiaType, no. 2017-PFS-24), Dutch Diabetes Research Fundation (project Innovate2CureType1, DDRF; no. 2018.10.002) and the Innovative Medicines Initiative 2 Joint Undertaking (project INNODIA, no. 115797). This Innovative Medicines Initiative 2 Joint Undertaking receives support from the EU's Horizon 2020 Research and Innovation Programme and European Federation of Pharmaceutical Industries and Associations, JDRF and the Leona M. and Harry B. Helmsley Charitable Trust (project INNODIA, no. 115797). T.O.M. and D.L.E. were supported by a grant from the National Institutes of Health-National Institute of Diabetes and Digestive and Kidney Diseases-Human Islet Research Network Consortium (no. 1UC4DK104166-01). Part of the work was performed at the Environmental Molecular Sciences Laboratory, a US Department of Energy national scientific user facility located at Pacific Northwest National Laboratory. Battelle operates the Pacific Northwest National Laboratory for the Department of Energy (contract no. DE-AC05-76RLO01830). We thank J. Mercader (Broad Institute) and M. Guindo Martínez (Barcelona Supercomputing Center) for helpful discussions regarding GWAS enrichment analyses and A. Castela (Université Libre de Bruxelles Center for Diabetes Research) for helping with the glucose-stimulated insulin secretion experiments.

\section{References:}

1. Todd JA Etiology of Type 1 Diabetes. Immunity 32, 457-467 (2010). [PubMed: 20412756]

2. Ziegler A-G \& Nepom GT Prediction and Pathogenesis in Type 1 Diabetes. Immunity 32, 468-478 (2010). [PubMed: 20412757]

3. Eizirik DL, Colli ML \& Ortis F The role of inflammation in insulitis and beta-cell loss in type 1 diabetes. Nat. Rev. Endocrinol 5, 219-26 (2009). [PubMed: 19352320]

4. Onengut-Gumuscu $\mathrm{S}$ et al. Fine mapping of type 1 diabetes susceptibility loci and evidence for colocalization of causal variants with lymphoid gene enhancers. Nat. Genet 47, 381-386 (2015). [PubMed: 25751624]

5. Farh KK et al. Genetic and epigenetic fine mapping of causal autoimmune disease variants. Nature 518, 337-343 (2015). [PubMed: 25363779]

6 . Ravassard $P$ et al. A genetically engineered human pancreatic $\beta$ cell line exhibiting glucoseinducible insulin secretion. J. Clin. Invest 121, 3589-3597 (2011). [PubMed: 21865645]

7. Ostuni R et al. Latent Enhancers Activated by Stimulation in Differentiated Cells. Cell 152, 157171 (2013). [PubMed: 23332752]

8. Gonzalez-Duque $S$ et al. Conventional and Neo-Antigenic Peptides Presented by $\beta$ Cells Are Targeted by Circulating Naïve CD8+ T Cells in Type 1 Diabetic and Healthy Donors. Cell Metab. (2018). doi:10.1016/j.cmet.2018.07.007

9. Pasquali $\mathrm{L}$ et al. Pancreatic islet enhancer clusters enriched in type 2 diabetes risk-associated variants. Nat. Genet 46, 136-143 (2014). [PubMed: 24413736]

10. Sung M-H, Guertin MJ, Baek S \& Hager GL DNase Footprint Signatures Are Dictated by Factor Dynamics and DNA Sequence. Mol. Cell 56, 275-285 (2014). [PubMed: 25242143]

11. Bird A DNA methylation patterns and epigenetic memory. Genes Dev. 16, 6-21 (2002). [PubMed: 11782440] 
12. Hemberger M, Dean W \& Reik W Epigenetic dynamics of stem cells and cell lineage commitment: digging Waddington's canal. Nat. Rev. Mol. Cell Biol 10, 526-537 (2009). [PubMed: 19603040]

13. Lister R et al. Human DNA methylomes at base resolution show widespread epigenomic differences. Nature 462, 315-322 (2009). [PubMed: 19829295]

14. Feinberg AP Phenotypic plasticity and the epigenetics of human disease. Nature 447, 433-440 (2007). [PubMed: 17522677]

15. Dixon JR et al. Topological domains in mammalian genomes identified by analysis of chromatin interactions. Nature 485, 376-380 (2012). [PubMed: 22495300]

16. Nora EP et al. Spatial partitioning of the regulatory landscape of the X-inactivation centre. Nature 485, 381-385 (2012). [PubMed: 22495304]

17. Dekker J, Marti-Renom MA \& Mirny LA Exploring the three-dimensional organization of genomes: interpreting chromatin interaction data. Nat. Rev. Genet 14, 390-403 (2013). [PubMed: 23657480]

18. Symmons $\mathrm{O}$ et al. Functional and topological characteristics of mammalian regulatory domains. Genome Res. 24, 390-400 (2014). [PubMed: 24398455]

19. Krivega I, Dale RK \& Dean A Role of LDB1 in the transition from chromatin looping to transcription activation. Genes Dev. 28, 1278-1290 (2014). [PubMed: 24874989]

20. Yu M \& Ren B The Three-Dimensional Organization of Mammalian Genomes. Annu. Rev. Cell Dev. Biol 33, 265-289 (2017). [PubMed: 28783961]

21. Rowley MJ \& Corces VG Organizational principles of 3D genome architecture. Nat. Rev. Genet 19, 789-800 (2018). [PubMed: 30367165]

22. Schoenfelder S \& Fraser P Long-range enhancer-promoter contacts in gene expression control. Nat. Rev. Genet 1 (2019). doi:10.1038/s41576-019-0128-0

23. Stadhouders R, Filion GJ \& Graf T Transcription factors and 3D genome conformation in cell-fate decisions. Nature 569, 345-354 (2019). [PubMed: 31092938]

24. Miguel-Escalada I et al. Human pancreatic islet three-dimensional chromatin architecture provides insights into the genetics of type 2 diabetes. Nat. Genet 51, 1137-1148 (2019). [PubMed: 31253982]

25. Schwartzman O et al. UMI-4C for quantitative and targeted chromosomal contact profiling. Nat. Methods 13, 685-691 (2016). [PubMed: 27376768]

26. MacArthur J et al. The new NHGRI-EBI Catalog of published genome-wide association studies (GWAS Catalog). Nucleic Acids Res. 45, D896-D901 (2017). [PubMed: 27899670]

27. Parker SCJ et al. Chromatin stretch enhancer states drive cell-specific gene regulation and harbor human disease risk variants. Proc. Natl. Acad. Sci 110, 17921-17926 (2013). [PubMed: 24127591]

28. Cooper $\mathrm{NJ}$ et al. Type 1 diabetes genome-wide association analysis with imputation identifies five new risk regions. bioRxiv 120022 (2017). doi:10.1101/120022

29. Fortune MD et al. Statistical colocalization of genetic risk variants for related autoimmune diseases in the context of common controls. Nat. Genet 47, 839-846 (2015). [PubMed: 26053495]

30. Ray A, Basu S, Williams CB, Salzman NH \& Dittel BN A Novel IL-10-Independent Regulatory Role for B Cells in Suppressing Autoimmunity by Maintenance of Regulatory T Cells via GITR Ligand. J. Immunol 188, 3188-3198 (2012). [PubMed: 22368274]

31. Davison LJ et al. Long-range DNA looping and gene expression analyses identify DEXI as an autoimmune disease candidate gene. Hum. Mol. Genet 21, 322-333 (2012). [PubMed: 21989056]

32. Dos Santos RS et al. DEXI, a candidate gene for type 1 diabetes, modulates rat and human pancreatic beta cell inflammation via regulation of the type I IFN/STAT signalling pathway. Diabetologia 62, 459-472 (2019). [PubMed: 30478640]

33. Alasoo $\mathrm{K}$ et al. Shared genetic effects on chromatin and gene expression indicate a role for enhancer priming in immune response. Nat. Genet 50, 424-431 (2018). [PubMed: 29379200]

34. Heinz $\mathrm{S}$ et al. Effect of natural genetic variation on enhancer selection and function. Nature 503, 487-492 (2013). [PubMed: 24121437] 
35. Vandenbon A, Kumagai Y, Lin M, Suzuki Y \& Nakai K Waves of chromatin modifications in mouse dendritic cells in response to LPS stimulation. Genome Biol. 19, 138 (2018). [PubMed: 30231913]

36. Phanstiel DH et al. Static and Dynamic DNA Loops form AP-1-Bound Activation Hubs during Macrophage Development. Mol. Cell 67, 1037-1048.e6 (2017). [PubMed: 28890333]

37. Mumbach MR et al. Enhancer connectome in primary human cells identifies target genes of disease-associated DNA elements. Nat. Genet 49, 1602-1612 (2017). [PubMed: 28945252]

38. Taberlay PC et al. Three-dimensional disorganization of the cancer genome occurs coincident with long-range genetic and epigenetic alterations. Genome Res. 26, 719-731 (2016). [PubMed: 27053337]

39. Barutcu AR et al. Chromatin interaction analysis reveals changes in small chromosome and telomere clustering between epithelial and breast cancer cells. Genome Biol. 16, 214 (2015). [PubMed: 26415882]

40. Chandra T et al. Global Reorganization of the Nuclear Landscape in Senescent Cells. Cell Rep. 10, 471-483 (2015). [PubMed: 25640177]

41. Criscione SW et al. Reorganization of chromosome architecture in replicative cellular senescence. Sci. Adv 2, e1500882 (2016). [PubMed: 26989773]

42. Le Dily F et al. Distinct structural transitions of chromatin topological domains correlate with coordinated hormone-induced gene regulation. Genes Dev. 28, 2151-2162 (2014). [PubMed: 25274727]

43. Op de Beeck A \& Eizirik DL Viral infections in type 1 diabetes mellitus - why the $\beta$ cells? Nat. Rev. Endocrinol 12, 263-273 (2016). [PubMed: 27020257]

44. Kim-Hellmuth $\mathrm{S}$ et al. Genetic regulatory effects modified by immune activation contribute to autoimmune disease associations. Nat. Commun 8, 266 (2017). [PubMed: 28814792]

\section{Methods-only References:}

45. Bucher P et al. Assessment of a Novel Two-Component Enzyme Preparation for Human Islet Isolation and Transplantation. Transplantation 79, 91-97 (2005). [PubMed: 15714175]

46. Melzi R et al. Role of CCL2/MCP-1 in Islet Transplantation. Cell Transplant. 19, 1031-1046 (2010). [PubMed: 20546673]

47. Eizirik DL et al. The Human Pancreatic Islet Transcriptome: Expression of Candidate Genes for Type 1 Diabetes and the Impact of Pro-Inflammatory Cytokines. PLoS Genet. 8, e1002552 (2012). [PubMed: 22412385]

48. Colli ML, Moore F, Gurzov EN, Ortis F \& Eizirik DL MDA5 and PTPN2, two candidate genes for type 1 diabetes, modify pancreatic $\beta$-cell responses to the viral by-product double-stranded RNA. Hum. Mol. Genet 19, 135-146 (2010). [PubMed: 19825843]

49. Ortis F et al. Cytokine-Induced Proapoptotic Gene Expression in Insulin-Producing Cells Is Related to Rapid, Sustained, and Nonoscillatory Nuclear Factor- $\kappa$ B Activation. Mol. Endocrinol 20, 1867-1879 (2006). [PubMed: 16556731]

50. Buenrostro JD, Giresi PG, Zaba LC, Chang HY \& Greenleaf WJ Transposition of native chromatin for fast and sensitive epigenomic profiling of open chromatin, DNA-binding proteins and nucleosome position. Nat. Methods 10, 1213-1218 (2013). [PubMed: 24097267]

51. Lavin Y et al. Tissue-Resident Macrophage Enhancer Landscapes Are Shaped by the Local Microenvironment. Cell 159, 1312-1326 (2014). [PubMed: 25480296]

52. Raurell-Vila H, Ramos-Rodríguez M \& Pasquali L Assay for Transposase Accessible Chromatin (ATAC-Seq) to Chart the Open Chromatin Landscape of Human Pancreatic Islets in Methods in Molecular Biology (eds. Vavouri T \& Peinado MA) 197-208 (Human Press, 2018). doi:10.1007/978-1-4939-7768-0_11

53. Schmidl C, Rendeiro AF, Sheffield NC \& Bock C ChIPmentation: fast, robust, low-input ChIP-seq for histones and transcription factors. Nat. Methods 12, 963-965 (2015). [PubMed: 26280331]

54. Langmead B \& Salzberg SL Fast gapped-read alignment with Bowtie 2. Nat. Methods 9, 357-359 (2012). [PubMed: 22388286] 
55. Dunhan I An integrated encyclopedia of DNA elements in the human genome. Nature 489, 57-74 (2012). [PubMed: 22955616]

56. Li H et al. The Sequence Alignment/Map format and SAMtools. Bioinformatics 25, 2078-2079 (2009). [PubMed: 19505943]

57. Zhang Y et al. Model-based Analysis of ChIP-Seq (MACS). Genome Biol. 9, R137 (2008). [PubMed: 18798982]

58. Juan-Mateu J et al. SRp55 Regulates a Splicing Network That Controls Human Pancreatic $\beta$-Cell Function and Survival. Diabetes 67, 423-436 (2018). [PubMed: 29246973]

59. Trapnell C, Pachter L \& Salzberg SL TopHat: discovering splice junctions with RNA-Seq. Bioinformatics 25, 1105-1111 (2009). [PubMed: 19289445]

60. Harrow J et al. GENCODE: The reference human genome annotation for The ENCODE Project. Genome Res. 22, 1760-1774 (2012). [PubMed: 22955987]

61. Anders S, Pyl PT \& Huber W HTSeq--a Python framework to work with high-throughput sequencing data. Bioinformatics 31, 166-169 (2015). [PubMed: 25260700]

62. Love MI, Huber W \& Anders S Moderated estimation of fold change and dispersion for RNA-seq data with DESeq2. Genome Biol. 15, 550 (2014). [PubMed: 25516281]

63. Nakayasu ES et al. MPLEx: a Robust and Universal Protocol for Single-Sample Integrative Proteomic, Metabolomic, and Lipidomic Analyses. mSystems 1, (2016).

64. Mayampurath AM et al. DeconMSn: a software tool for accurate parent ion monoisotopic mass determination for tandem mass spectra. Bioinformatics 24, 1021-1023 (2008). [PubMed: 18304935]

65. Petyuk VA et al. DtaRefinery, a Software Tool for Elimination of Systematic Errors from Parent Ion Mass Measurements in Tandem Mass Spectra Data Sets. Mol. Cell. Proteomics 9, 486-496 (2010). [PubMed: 20019053]

66. Kim S \& Pevzner PA MS-GF+ makes progress towards a universal database search tool for proteomics. Nat. Commun 5, 5277 (2014). [PubMed: 25358478]

67. Webb-Robertson B-JM et al. Bayesian Proteoform Modeling Improves Protein Quantification of Global Proteomic Measurements. Mol. Cell. Proteomics 13, 3639-3646 (2014). [PubMed: 25433089]

68. Li T et al. GeNets: a unified web platform for network-based genomic analyses. Nat. Methods 15, 543-546 (2018). [PubMed: 29915188]

69. Li T et al. A scored human protein-protein interaction network to catalyze genomic interpretation. Nat. Methods 14, 61-64 (2017). [PubMed: 27892958]

70. Herwig R, Hardt C, Lienhard M \& Kamburov A Analyzing and interpreting genome data at the network level with ConsensusPathDB. Nat. Protoc 11, 1889-1907 (2016). [PubMed: 27606777]

71. Liberzon A et al. Molecular signatures database (MSigDB) 3.0. Bioinformatics 27, 1739-1740 (2011). [PubMed: 21546393]

72. Heinz S et al. Simple Combinations of Lineage-Determining Transcription Factors Prime cisRegulatory Elements Required for Macrophage and B Cell Identities. Mol. Cell 38, 576-589 (2010). [PubMed: 20513432]

73. Wickham H Ggplot2 : elegant graphics for data analysis. (Springer, 2009).

74. Moran S, Arribas C \& Esteller M Validation of a DNA methylation microarray for $850,000 \mathrm{CpG}$ sites of the human genome enriched in enhancer sequences. Epigenomics 8, 389-399 (2016). [PubMed: 26673039]

75. Assenov $\mathrm{Y}$ et al. Comprehensive analysis of DNA methylation data with RnBeads. Nat. Methods 11, 1138-1140 (2014). [PubMed: 25262207]

76. Ritchie ME et al. limma powers differential expression analyses for RNA-sequencing and microarray studies. Nucleic Acids Res. 43, e47-e47 (2015). [PubMed: 25605792]

77. Du P et al. Comparison of Beta-value and M-value methods for quantifying methylation levels by microarray analysis. BMC Bioinformatics 11, 587 (2010). [PubMed: 21118553]

78. Aronesty E Comparison of Sequencing Utility Programs. Open Bioinforma. J 7, 1-8 (2013).

79. Ahmed M et al. Variant Set Enrichment: an R package to identify disease-associated functional genomic regions. BioData Min. 10, 9 (2017). [PubMed: 28239419] 
80. Gibbs RA et al. A global reference for human genetic variation. Nature 526, 68-74 (2015). [PubMed: 26432245]

81. Jorda M Upregulation of MMP-9 in MDCK epithelial cell line in response to expression of the Snail transcription factor. J. Cell Sci 118, 3371-3385 (2005). [PubMed: 16079281]

82. Brozzi $\mathrm{F}$ et al. Cytokines induce endoplasmic reticulum stress in human, rat and mouse beta cells via different mechanisms. Diabetologia 58, 2307-2316 (2015). [PubMed: 26099855]

83. Mularoni L, Ramos-Rodríguez M \& Pasquali L The pancreatic islet regulome browser. Front. Genet 8, 1-8 (2017). [PubMed: 28179914] 
a
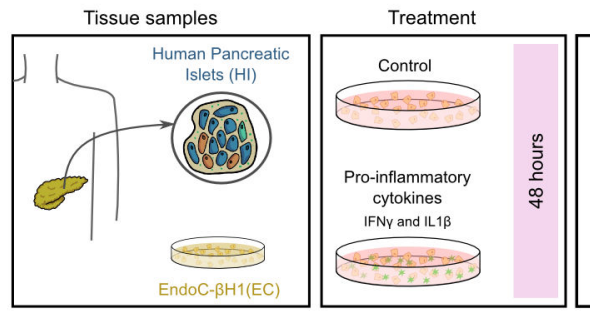

c
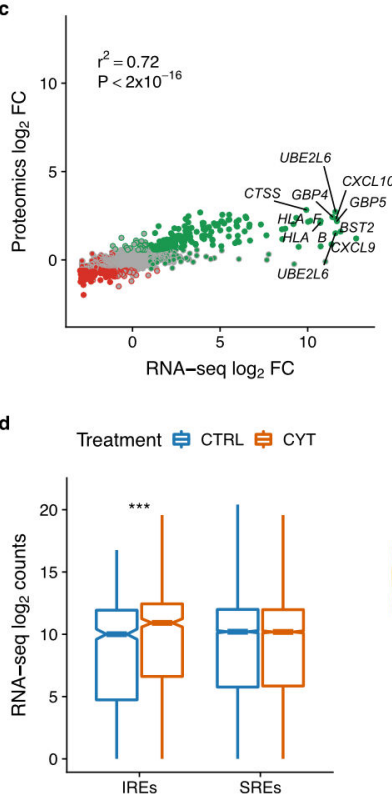
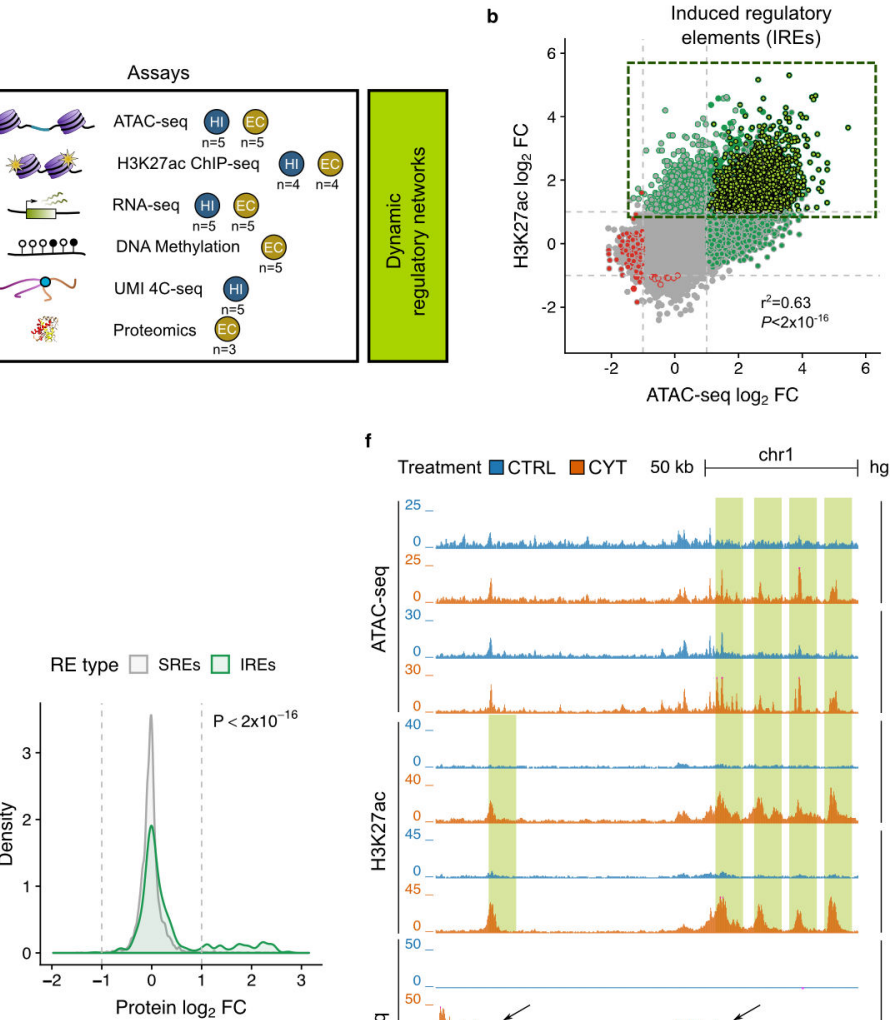

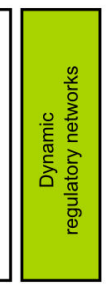

Treatment $\square$ CTRL $\square$ CYT $50 \mathrm{~kb} \longmapsto \mathrm{chr} 1 \quad \mid$ hg 19

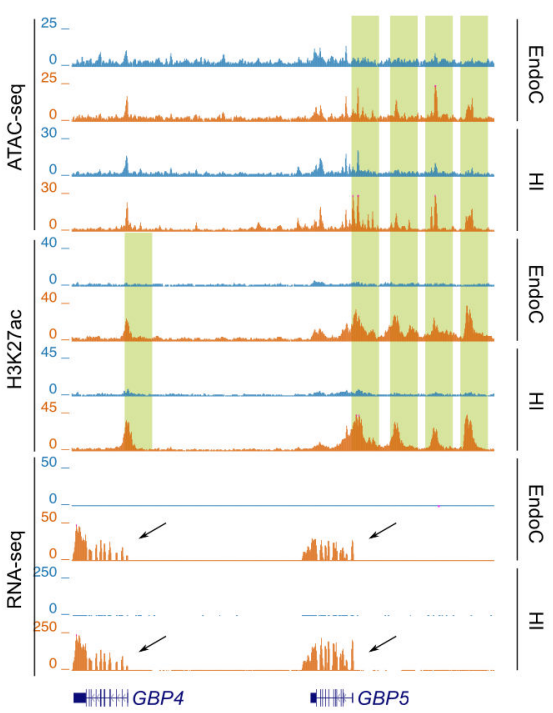

Figure 1. Pro-inflammatory cytokines exposure causes profound remodeling of the $\beta$-cells regulatory landscape.

a, Summary of the experimental design. The number of EndoC- $\beta$ H1 (EC) and human pancreatic islets (HI) samples used in different assays is indicated. b, Correlation between chromatin accessibility and $\mathrm{H} 3 \mathrm{~K} 27$ ac deposition, each dot corresponds to a chromatin site. The point fill refers to the ATAC-seq and the border to the H3K27ac classification (gained=green; lost=red; stable=grey). The dotted box depicts the regulatory elements referred as induced regulatory elements (IREs) and the lighter shade of green depicts a subtype named neo IREs (see text). c, Correlation between changes in RNA expression and protein abundance in EndoC- $\beta \mathrm{H} 1$ cells. Point fill and border indicate the classification of RNA-seq and protein respectively (up-regulated=green; down-regulated=red; equalregulated=grey). $\mathbf{d}$, Genes proximal to IREs (see methods) show cytokine-induced expression in EndoC- $\beta \mathrm{H} 1$ exposed or not to pro-inflammatory treatment. CYT=cytokine exposed, CTRL=control. ***Two-sided Wilcoxon test $P<0.001$. Box plot limits show upper and lower quartiles, whiskers extend to 1.5 times the interquartile range. e, Translation of proteins encoded by IRE-associated genes is induced by cytokine exposure in EndoC- $\beta \mathrm{H} 1$. This is shown by the significantly different (Two-sided Wilcoxon test $P<2 \times 10^{-16}$ ) $\log _{2}$ FC distribution of protein abundance obtained after cytokine exposure, for proteins encoded by genes associated with IREs or stable regulatory elements (SREs). f, Representative view of 
the IFN-inducible guanylate binding proteins $G B P 4$ and $G B P 5$, illustrating their upregulation upon cytokine exposure and the nearby induction of IREs characterized by gains in chromatin accessibility and enrichment in H3K27ac (green boxes). 

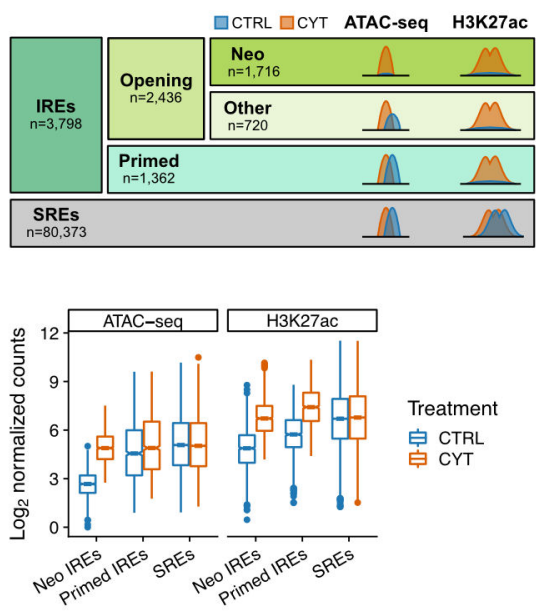

b

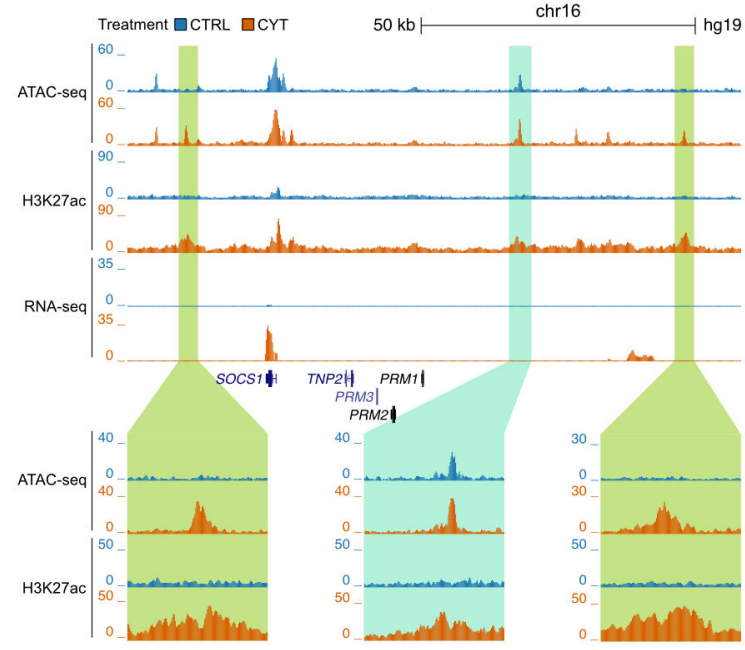

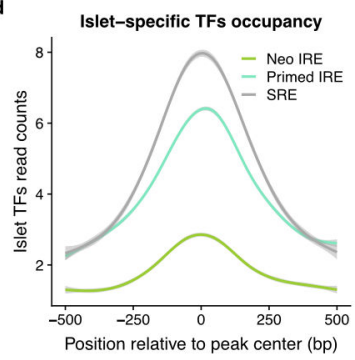

e Neo enhancers ISRE

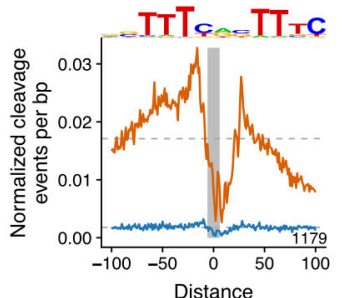

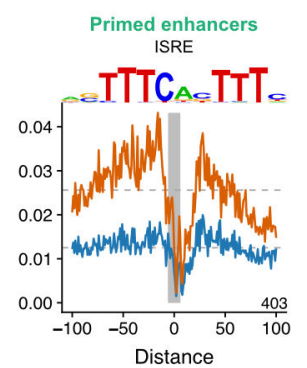

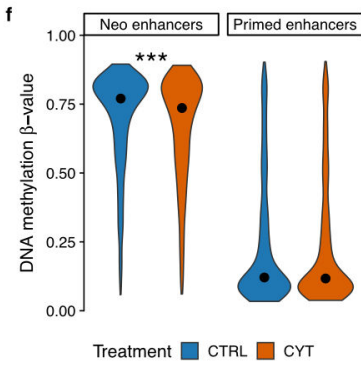

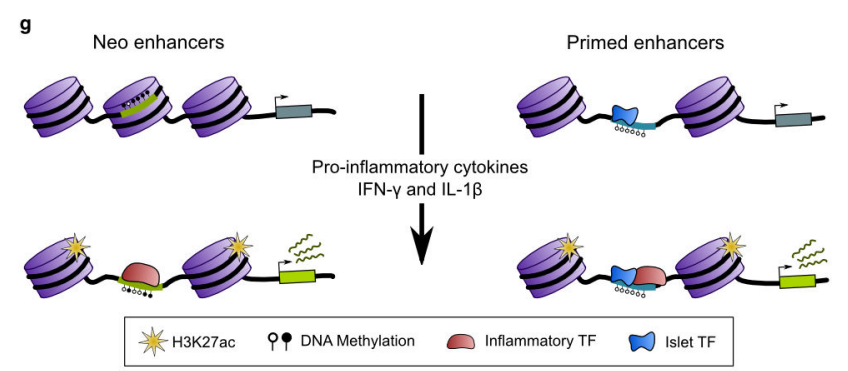

Figure 2. The $\beta$ cell response to pro-inflammatory cytokine unveils neo and primed regulatory elements.

a, Classification of ATAC-seq open chromatin sites upon exposure of human $\beta$ cells to IFN$\gamma$ and IL-1 $\beta$. IREs=induced regulatory elements, SREs=stable regulatory elements. $\mathbf{b}$, View of the SOCS1 locus, a gene strongly induced upon pro-inflammatory cytokine exposure. We here depict representative examples of primed (blue box) and neo IREs (green boxes). c, Box plot distribution of ATAC-seq and H3K27ac normalized tag counts at different classes of IREs. Box plot limits show upper and lower quartiles, whiskers extend to 1.5 times the interquartile range, individual data points represent outliers and the notch represents the confidence interval around the median. d, Islet-specific TF occupancy at neo, primed and stable regulatory elements. Read density for PDX1, NKX2.2, FOXA2, NKX6.1 and MAFB was calculated in $10 \mathrm{bp}$ bins in $1 \mathrm{~kb}$ windows centered on the regulatory element. Lines represent means, while the grey shades depict the standard deviation. e, Footprint analysis of ISRE motifs in neo (left) and primed regulatory elements (right) in cells exposed or not to IFN- $\gamma$ and IL-1 $\beta$ (control = blue; cytokines = orange). $\mathbf{f}$, Violin plots showing the 
distribution of DNA methylation $\beta$-values in neo and primed enhancers, exposed or not to pro-inflammatory cytokines. *** Two-sided Wilcoxon test $P<0.001$. g, Model showing two types of IREs driving the response to pro-inflammatory cytokines in human $\beta$ cells. 

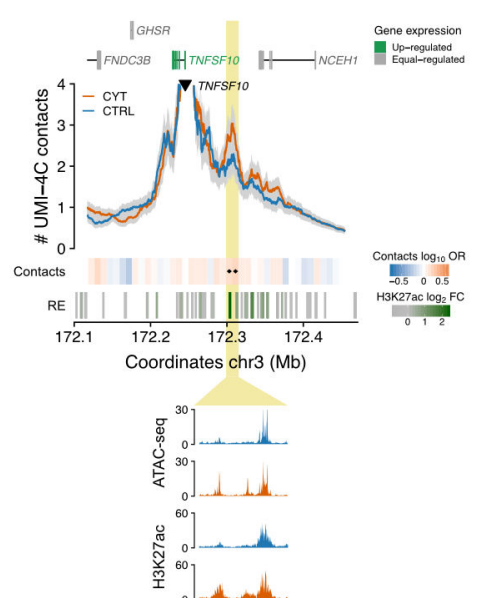

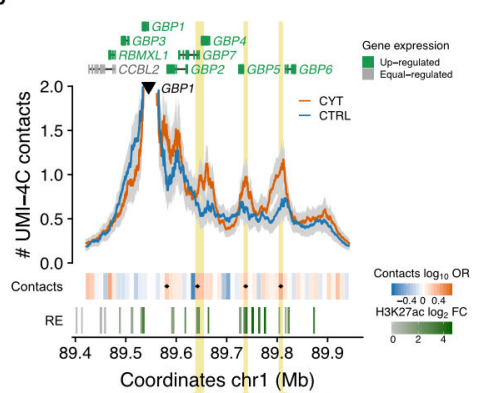

Coordinates chr1 $(\mathrm{Mb})$

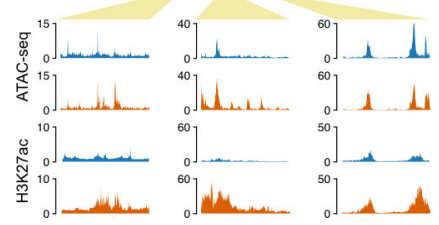

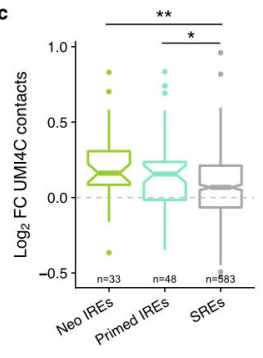

Figure 3. Cytokine exposure induces changes in human islet 3D chromatin structure.

$\mathbf{a}, \mathbf{b}$, View of the UMI-4C chromatin contacts of $T N F S F 1 O(\mathbf{a})$ and $G B P 1(\mathbf{b})$ promoters, before and after exposure to pro-inflammatory cytokines. Yellow boxes indicate IREs that gain contacts with the up-regulated gene promoters. A heatmap under the $4 \mathrm{C}$ track represents the $\log _{10}$ odds ratio (OR) of the UMI-4C contacts difference in cytokine vs. control. Small black diamonds on top of the contact heatmap indicate a significant difference between cytokine-treated and control samples 3D chromatin contacts (Chi-squared $P<0.05$ ). ATAC-seq peaks are represented by rectangles shaded from gray to green proportionally to the cytokine-induced $\mathrm{H} 2 \mathrm{~K} 27 \mathrm{ac} \log _{2}$ fold change observed at that site (RE=regulatory elements track). c, Distribution of the UMI-4C contacts $\log _{2}$ fold changes (cytokines vs. control) at the different types of islets open chromatin sites classified as in Fig. 2a. The data, obtained by analyzing viewpoints centered at the promoter of cytokine-induced genes, show that the chromatin structural changes are preferentially happening at IREs. Box plot limits show upper and lower quartiles, whiskers extend to 1.5 times the interquartile range, individual data points represent outliers and the notch represents the confidence interval around the median. Two-sided Wilcoxon test $* P<0.05$, ** $P<0.01$, *** $P<0.001$. 


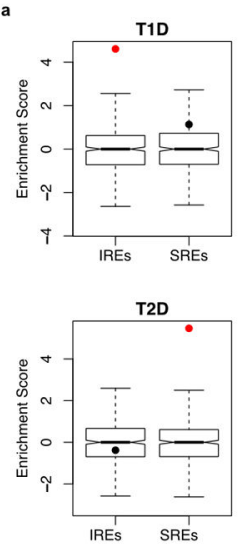

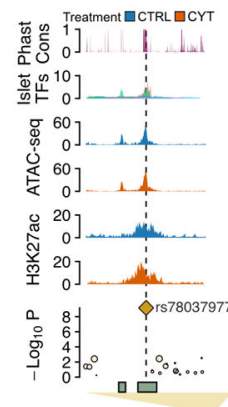

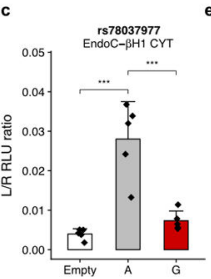

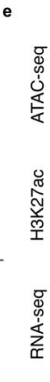
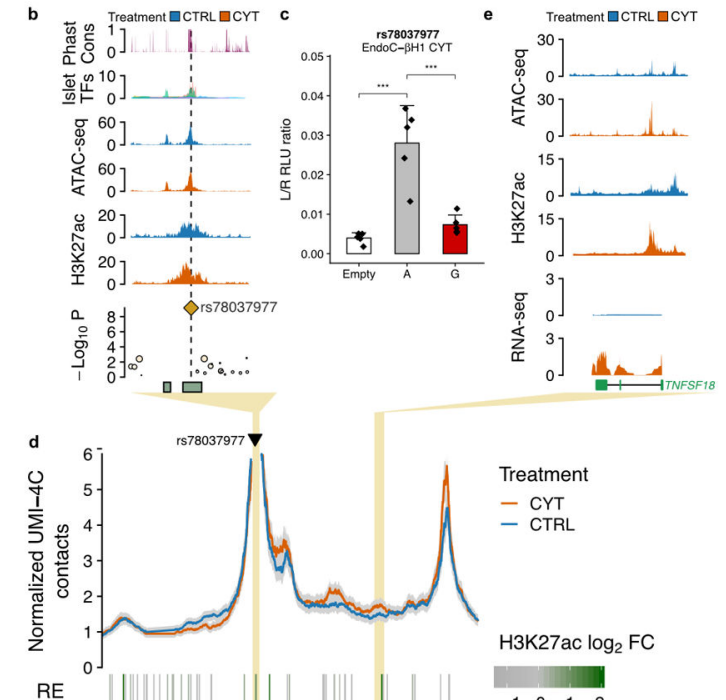

$\mathrm{H} 3 \mathrm{~K} 27 \mathrm{ac} \log _{2} \mathrm{FC}$

$\begin{array}{llll}-1 & 0 & 1 & 2\end{array}$

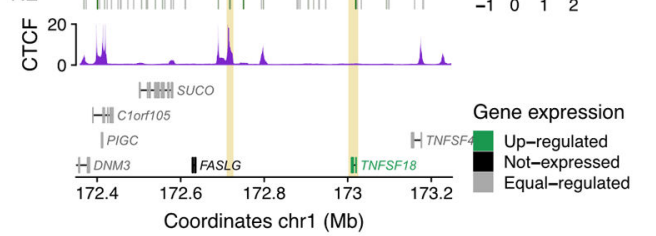

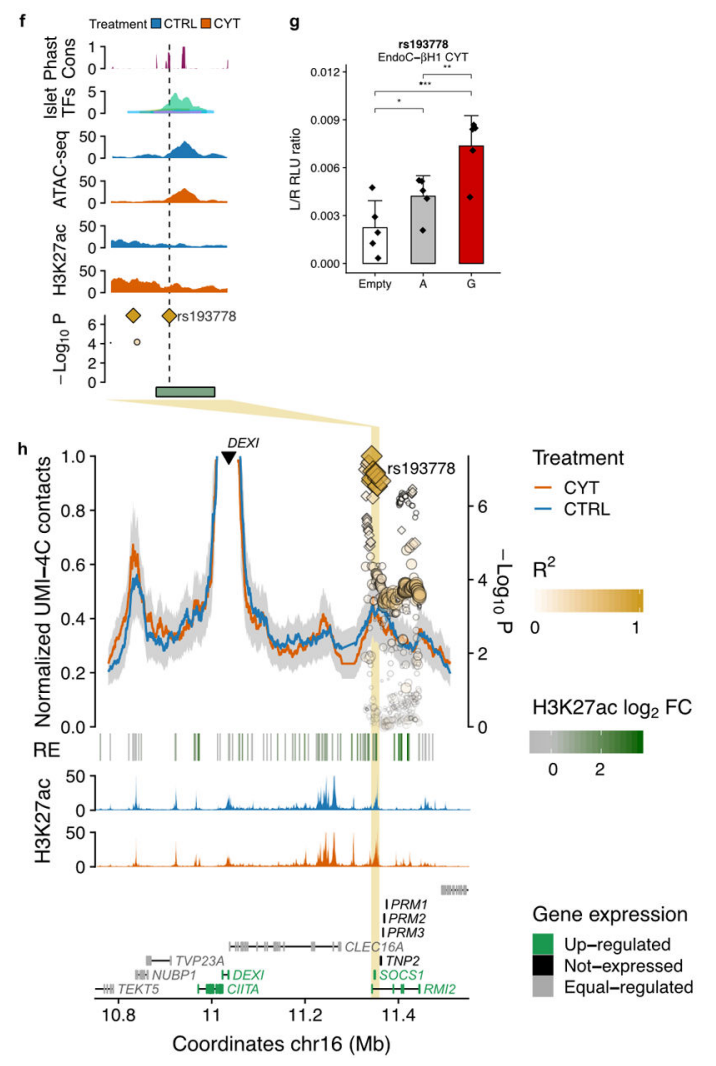

Figure 4. Cytokine-induced islet regulatory elements map to T1D associated regions and guide the identification of functional risk variants.

a, Islet IREs are enriched for T1D but not T2D risk variants while the opposite is true for islet SREs. Significant Varian Set Enrichment (VSE) scores are depicted in red (Bonferroni adjusted $P<0.05$ ). Box plot limits show upper and lower quartiles, whiskers extend to 1.5 times the interquartile range and notch represents the median confidence interval for distributions of matched null sets (500 permutations). b, rs78037977 overlaps an IRE bound by islet-specific TFs under basal conditions. c, Luciferase assays in EndoC- $\beta \mathrm{H} 1$ exposed to cytokines show that the sequence exerts enhancer activity which is reduced in the T1D associated allele (G). d, UMI-4C in human islets show that the IRE containing rs78037977 engages multiple distal chromatin contacts. e, Zoom-in at one induced chromatin contact, mapping to the up-regulated TNFSF18 gene. f, Variant rs193778 maps to a phylogenetically conserved IRE. g, Luciferase assays in EndoC- $\beta \mathrm{H} 1$ exposed to cytokines show significantly increased enhancer activity of the risk (G) allele compared to the non-risk (A) allele. $\mathbf{h}$, UMI-4C in islets using the promoter of $D E X I$ as viewpoint, show a chromatin contact with the IRE bearing the T1D risk variant. ATAC-seq peaks are represented, in $\mathbf{d}$ and $\mathbf{h}$, by rectangles shaded proportionally to the $\mathrm{H} 2 \mathrm{~K} 27 \mathrm{ac} \log _{2}$ fold change ( $\mathrm{RE}=$ regulatory elements). Statistical significance in $\mathbf{c}$ and $\mathbf{g}$ was determined by one-way ANOVA tests followed by Bonferroni's correction. ${ }^{*} P<0.05, * * P<0.01, * * * P<0.001$. Bars represent mean \pm sd. 